\title{
Empirische Kapitalmarktforschung zu Conference Calls: Eine Literaturanalyse
}

\author{
Devrimi Kaya (D) Christian Maier • Tobias Böhmer
}

Eingegangen: 2. Februar 2018 / Angenommen: 31. Dezember 2019 / Online publiziert: 28. Januar 2020 (C) Der/die Autor(en) 2020

Zusammenfassung Conference Calls im Rahmen der Ergebnisberichterstattung haben sich in den vergangenen Jahrzehnten zu einem wichtigen Instrument der Offenlegung an Kapitalmärkten entwickelt. Dieser Beitrag analysiert die empirische Literatur zu den Determinanten und ökonomischen Konsequenzen von Conference Calls. Wir strukturieren unsere Literaturanalyse anhand von Themensträngen wie dem Zugang zu Conference Calls, der Teilnehmerpartizipation und den Kapitalmarktreaktionen. Zudem werden Studien analysiert, die das Phänomen Conference Call untersuchen, um wichtige Einblicke in den Informationsgehalt von Conference Calls und das Kommunikationsverhalten der Teilnehmer zu erhalten. Abschließend werden Anregungen für künftige Forschungsarbeiten gegeben. Gegenstand der Literaturanalyse bilden 49 Beiträge in international renommierten Zeitschriften aus den Jahren 1998 bis 2018.

Schlüsselwörter Conference Calls · Kapitalmarktkommunikation · Ergebnisbezogene Analystenkonferenz $\cdot$ Literaturanalyse

JEL-codes M10 $\cdot$ M41 $\cdot$ M48 $\cdot$ G10 $\cdot$ G30

Zusatzmaterial online Zusätzliche Informationen sind in der Online-Version dieses Artikels (https:// doi.org/10.1007/s41471-019-00080-2) enthalten.

D. Kaya $(\bowtie) \cdot$ T. Böhmer

Lehrstuhl für Corporate Governance, Auditing und Accounting, Ruhr-Universität Bochum, Universitätsstr. 150, 44801 Bochum, Deutschland

E-Mail: devrimi.kaya@rub.de

T. Böhmer

E-Mail: tobias.boehmer@rub.de

C. Maier

Rödl \& Partner GmbH Wirtschaftsprüfungsgesellschaft Steuerberatungsgesellschaft,

Äußere Sulzbacher Straße 100, 90491 Nürnberg, Deutschland

E-Mail: christian.maier@ roedl.de 


\title{
Empirical Capital Market Research on Conference Calls: A Literature Review
}

\begin{abstract}
Over the last decades, conference calls held in conjunction with an earnings release have become an important instrument for disclosure on capital markets. This paper reviews the empirical literature on the determinants and economic consequences of conference calls. We structure our literature review around topics such as the access to conference calls, the participation and capital market reactions. In addition, we review studies, which examine the phenomenon conference call to gain important insights into the information content of conference calls as well as the communication behavior of participants. Finally, our review identifies opportunities for future research. The literature review includes 49 studies from highly-ranked international journals between 1998 and 2018.
\end{abstract}

Keywords Conference calls · Capital market communication · Earnings call · Literature review

\section{Einführung}

Ergebnisorientierte Conference Calls werden von Unternehmen als Instrument eingesetzt, um den Kapitalmarkt gezielt mit Informationen zu versorgen. Diese bestehen in der Regel aus einem Präsentationsteil, in dem das Management über die zuletzt veröffentlichten Quartalszahlen und prognostizierte Finanzergebnisse berichtet, und einem anschließenden Diskussionsteil (auch als Q\&A bezeichnet), in dem die Teilnehmer die Gelegenheit erhalten, Fragen direkt an das Management zu stellen (Böhmer et al. 2019). Conference Calls ermöglichen im Vergleich zur regulierten Finanzberichterstattung eine freiere und interaktivere Kommunikation sowie einen einzigartigen Einblick in die spezifischen Informationsbedürfnisse von Analysten und Investoren. Dabei ist in der Forschungsliteratur nicht abschließend geklärt, ob Conference Calls eher eine substituierende oder komplementäre Rolle gegenüber der klassischen Finanzberichterstattung zukommt. ${ }^{1}$ Seit nunmehr 20 Jahren wird die Kommunikation zwischen Unternehmen und dem Kapitalmarkt in Form von Conference Calls empirisch untersucht. Die große Zahl von aktuellen Beiträgen in der Literatur sowie die unterschiedliche inhaltliche und methodische Ausrichtung machen eine Bestandsaufnahme der Forschungsergebnisse sinnvoll.

Der vorliegende Beitrag analysiert systematisch die Forschungsliteratur zu Conference Calls. Gegenstand der Literaturanalyse bilden 49 empirische Arbeiten, die in führenden Fachzeitschriften im Zeitraum von 1998 bis 2018 publiziert wurden. Zunächst werden Beiträge analysiert, welche das Phänomen Conference Call als Offenlegungsinstrument explizit untersuchen. Das Hauptaugenmerk dieser Studien liegt auf den Charakteristika von Conference Calls wie etwa deren Umfang oder

\footnotetext{
1 Die bisherige Literatur ist uneins darüber, ob es sich bei Conference Calls um grundlegend neue, erweiternde Informationen handelt oder ob diese die bereits öffentlich zugänglichen Informationen nur weiter erläutern bzw. andere Kommunikationskanäle ersetzen (Bowen et al. 2002; Bassemir et al. 2013).
} 
Informationsgehalt. Die hohe Relevanz der Studien etwa zur Messung des Informationsgehalts zeigt sich darin, dass 22 von 25 publizierten Studien ab dem Jahr 2014 computerlinguistische Verfahren zur strukturierten Inhaltsanalyse von Conference Call Protokollen verwenden. In der Vergangenheit war es schwierig, die qualitativen Inhalte zu extrahieren und zu analysieren. Das neue Forschungsfeld der computergestützten Textanalyse erlaubt es z. B. das Sentiment von Conference Call Protokollen zu analysieren oder eine differenzierte Unterscheidung der Komplexität eines Textes in Zusatzinformation (latent information) und Verschleierung (obfuscation) vorzunehmen.

Neben dem Informationsgehalt von Conference Calls können auch wichtige Einblicke in das Kommunikationsverhalten des Managements gegenüber Finanzanalysten gewonnen werden und inwiefern das Management beispielsweise die Offenlegung von wettbewerbssensiblen Informationen vermeidet. Diese Erkenntnisse sollten größtenteils unabhängig vom Rechtsrahmen und der Regulierung der Ausrichtungsform von Conference Calls auch für eine deutsche Leserschaft von hohem Interesse sein.

Der Hauptfokus der Literaturanalyse liegt auf der empirischen Evidenz zu den Determinanten und ökonomischen Wirkungen von Conference Calls. Hierzu gehören beispielsweise Studien, welche die Ausgestaltungsform von Conference Calls und die daraus folgenden Kapitalmarktreaktionen untersuchen. Während im US-amerikanischen Setting seit der Regulation Fair Disclosure (Reg FD) im Jahr 2000 die offene Ausrichtung von Conference Calls verpflichtend vorgeschrieben ist, können sich kapitalmarktorientierte Unternehmen in Deutschland zwischen einer offenen und geschlossenen Ausrichtung entscheiden. Daher ist es wichtig, die Determinanten solcher Ausübungsformen zu kennen und deren ökonomische Wirkungen zu verstehen. Beispielsweise zeigen Studien, dass die offene Gestaltung von Conference Calls als eine wesentliche Informationsquelle von kleineren Investoren dienen kann und diese infolgedessen ihre Handelsaktivitäten verstärken (Bushee et al. 2004; Lee et al. 2004). Somit können insbesondere frühere US-Studien vor Reg FD dem deutschen bzw. europäischen Gesetzgeber nützliche Informationen für die künftige Regulierung des Offenlegungsinstruments geben. Gleichzeitig können jedoch auch Studien nach Reg FD im Hinblick auf die steigende Anzahl der offenen Conference Calls in Deutschland wertvolle Anhaltspunkte für die Kommunikation ohne selektive Informationsweitergabe liefern. ${ }^{2}$

Gleichzeitig entwickelt sich das Forschungsfeld in den letzten Jahren stark weiter. So sind beispielsweise neue, interdisziplinäre Forschungsstränge wie Persönlichkeitsmerkmale des Managements (u. a. Narzissmus) im Rahmen der Conference Call Literatur festzustellen. Somit ergeben sich zahlreiche Möglichkeiten zur Beantwortung offener Forschungsfragen, welche in Unterabschnitten identifiziert werden. Aufgrund der starken Fokussierung der bisherigen Forschung auf den US-Markt, sehen wir wie auch Bassemir et al. (2013) nicht zwangsläufig eine direkte Übertrag-

\footnotetext{
${ }^{2}$ Wir weisen jedoch darauf hin, dass eine offene Gestaltung von Conference Calls die selektive Offenlegung zwar einschränkt, aber nicht komplett ausschließen kann. So kann das Management einzelnen Analysten sowie Investoren nach Ausrichtung eines Conference Calls zusätzlich individuelle Treffen einräumen.
} 
barkeit der Ergebnisse auf den deutschen Rechtsraum gegeben. Trotz Unterschieden in der Corporate Governance und Kapitalmarktentwicklung gegenüber dem US-amerikanischen Rechtsraum gibt es jedoch keine Indizien dafür, dass sich die Ergebnisse in anderen Rechtsräumen fundamental ändern könnten. ${ }^{3}$

Unsere Literaturanalyse trägt auf unterschiedliche Weise zur Rechnungslegungsforschung bei. Im Besonderen erweitern wir bisherige Literaturanalysen im Offenlegungs- und Informationsumfeld von Unternehmen. Während die bisherige Literatur die ökonomischen Konsequenzen der Regulierung der traditionellen Finanzberichterstattung (Leuz und Wysocki 2016; Minnis und Shroff 2017), die Informationsverarbeitung einzelner Kapitalmarktakteure wie Analysten (Bradshaw 2011) oder Aspekte der freiwilligen und verpflichtenden Offenlegung im Informationsumfeld (Beyer et al. 2010) untersucht, analysiert unsere Studie als erste systematisch und umfassend das Forschungsfeld Conference Calls. Unser Rahmenkonzept umfasst neben den Determinanten und ökonomischen Konsequenzen auch eine detaillierte Untersuchung des Phänomens Conference Call als Offenlegungsinstrument. Durch das interaktive Format von Conference Calls lassen sich direkte Rückschlüsse auf die an der Kapitalmarktkommunikation beteiligten Individuen ziehen, die durch andere Kommunikationskanäle (wie etwa die formale, schriftliche Finanzberichterstattung) nicht identifiziert werden können. Beispielsweise gewähren Conference Calls exklusive Einblicke in die Informationsbeschaffung und -verarbeitung von Finanzanalysten. Diese Erkenntnisse können Literaturanalysen über Analysten als Kapitalmarktteilnehmer um aktuelle Aspekte ergänzen (z.B. Bradshaw 2011) und wichtige Hinweise darauf geben, wie Unternehmen ihr Kommunikationsverhalten im Rahmen von Conference Calls ausgestalten.

Des Weiteren bieten die Erkenntnisse der vorgestellten Studien wichtige Anhaltspunkte für deutsche Unternehmen und Rechnungslegungsadressaten. Gerade vor dem Hintergrund der zunehmenden Relevanz und Verbreitung des Mediums Conference Calls in Deutschland (siehe Tab. 1) ist eine Analyse der bisherigen Erkenntnisse und der vielfältigen ökonomischen Konsequenzen sinnvoll. Auch für den deutschen bzw. europäischen Gesetzgeber kann dieser Beitrag nützliche Informationen liefern. So verdeutlicht die Analyse der bisherigen Literatur die Wirkungsweise von selektiver Offenlegung. Folglich können die Erkenntnisse zur Debatte um die Schaffung eines Level Playing Fields zwischen verschiedenen Kapitalmarktteilnehmern beitragen und Hinweise für (zukünftige) regulatorische Eingriffe geben. Zudem informiert der Beitrag die Leserschaft über aktuelle Forschungsstränge und identifiziert neue Forschungsfragen. Darüber hinaus werden neue Verfahren der computergestützten Textanalyse aufgezeigt, welche zur methodischen Weiterentwicklung des Forschungsfelds beitragen und die empirische Kapitalmarktforschung zukünftig noch stärker prägen werden.

Der Beitrag ist wie folgt aufgebaut: Im zweiten Abschnitt werden die Grundlagen und institutionellen Rahmenbedingungen zu Conference Calls dargestellt. AnschlieBend folgt in Abschn. 3 die Auswahl der im Rahmen dieser Arbeit untersuchten Beiträge. Die ausgewählten Forschungsarbeiten werden in Abschn. 4 anhand des

\footnotetext{
${ }^{3}$ Für den deutschen Rechtsraum weisen beispielsweise Bassemir et al. (2013) Ergebnisse auf, die mit der US-Literatur (vor Reg FD) weitgehend konsistent sind.
} 
Rahmenkonzepts abgegrenzt und diskutiert. Abschließend fasst Abschn. 5 die wesentlichen Erkenntnisse zusammen.

\section{Begriffsdefinition und Institutionelle Rahmenbedingungen}

Der Begriff „Conference Call““ ist nicht eindeutig definiert. In dieser Arbeit werden unter Conference Calls Veranstaltungen verstanden, die vom Top-Management ausgerichtet werden, überwiegend an Analysten aber auch Investoren gerichtet sind und in Form von Telefonkonferenzen, Webkonferenzen oder physischen Veranstaltungen mit Telefon- oder Webübertragung stattfinden. Nicht darunter fallen Tagesveranstaltungen für Analysten und Investoren wie etwa Investorentage oder Capital Market Days. Beispielsweise sind nach $§ 55$ der Börsenordnung der Frankfurter Wertpapierbörse Aktienemittenten des Prime Standards dazu verpflichtet, mindestens einmal jährlich außerhalb der Bilanzpressekonferenz eine Analystenveranstaltung durchzuführen. Über eine bestimmte Form der Ausgestaltung gibt es keine konkreten Angaben. Dieser Beitrag fokussiert sich auf die empirische Evidenz von jenen Conference Calls, die im Rahmen der Ergebnisberichterstattung durchgeführt werden (sog. earnings calls).

Bei Conference Calls wird nach Zugang und Art der Teilnahme unterschieden. Unter geschlossenen Conference Calls werden dabei solche Veranstaltungen verstanden, deren Zugang auf eingeladene Teilnehmer begrenzt ist (Bushee et al. 2003). Conference Calls, bei denen zumindest eine passive Teilnahme für jeden Marktteilnehmer möglich ist, werden als offene Conference Calls bezeichnet. Im Unterschied zu den USA existiert in Deutschland keine Verpflichtung zur offenen Ausrichtung. Im Deutschen Corporate Governance Kodex wird lediglich auf die Gleichbehandlung der Aktionäre unter gleichen Voraussetzungen hingewiesen, sodass Aktionären alle wesentlichen neuen Tatsachen, die beispielsweise Finanzanalysten mitgeteilt wurden, ebenfalls zur Verfügung gestellt werden sollen (Grundsatz 20 DCGK).

Tab. 1 zeigt die Verbreitung von ergebnisorientierten Conference Calls bei Unternehmen des DAX, MDAX und SDAX für die Jahre 2010 und 2018. Die deskriptive

Tab. 1 Verbreitung von ergebnisorientierten Conference Calls in Deutschland (4. Quartal)

\begin{tabular}{lllll}
\hline & $\begin{array}{l}\text { Anzahl } \\
\text { Unternehmen }\end{array}$ & $\begin{array}{l}\text { Anzahl Conference } \\
\text { Calls }\end{array}$ & $\begin{array}{l}\text { Offene Conference } \\
\text { Calls }\end{array}$ & $\begin{array}{l}\text { Geschlossene Confe- } \\
\text { rence Calls }\end{array}$ \\
\hline 2010 & & & $25(83,3 \%)$ & $5(16,7 \%)$ \\
DAX & 30 & $30(100,0 \%)$ & $26(60,5 \%)$ & $17(39,5 \%)$ \\
MDAX & 50 & $43(86,0 \%)$ & $14(53,8 \%)$ & $12(46,6 \%)$ \\
SDAX & 50 & $26(52,0 \%)$ & $65(65,7 \%)$ & $34(34,3 \%)$ \\
Gesamt & 130 & $99(76,2 \%)$ & & $1(3,3 \%)$ \\
2018 & & $30(100,0 \%)$ & $29(96,7 \%)$ & $12(21,1 \%)$ \\
DAX & 30 & $57(95,0 \%)$ & $45(78,9 \%)$ & $28(45,9 \%)$ \\
MDAX & 60 & $61(87,1 \%)$ & $33(54,1 \%)$ & $41(27,7 \%)$ \\
SDAX & 70 & $148(92,5 \%)$ & $107(72,3 \%)$ & \\
Gesamt & 160 & & &
\end{tabular}

Eigene Erhebung auf Basis der Unternehmenswebseiten und Thomson Reuters StreetEvents 
Analyse zeigt, dass die Anzahl von Conference Calls im Zeitablauf steigt. So führen ca. $93 \%$ der Unternehmen einen Conference Call zum Jahresende 2018 durch. Hinsichtlich des Zugangs entscheiden sich im Zeitablauf mehr Unternehmen für eine offene Durchführung. Zudem zeigt sich, dass insbesondere Unternehmen aus dem MDAX bzw. SDAX den Zugang zu ihren Conference Calls heterogen ausgestalten. Die in der folgenden Literaturanalyse gewonnenen Erkenntnisse zur unterschiedlichen Ausrichtungsform von Conference Calls können daher für Aufsichtsbehörden interessant sein und zur Debatte über einen gleichberechtigten Informationszugang für alle Marktteilnehmer beitragen.

\section{Auswahl relevanter Untersuchungen}

Zunächst ist eine sinnvolle Auswahl der zu untersuchenden Forschungsarbeiten zu treffen. Hierzu erfolgt auf Basis des VHB-JOURQUAL3 aus dem Jahr 2015 eine Auswahl aller A+, A und B Journals aus den Bereichen „Rechnungswesen“ und „Bankbetriebslehre/Finanzierung“. ${ }^{4}$ Die Auswahl ergibt 35 Zeitschriften aus dem Bereich „Rechnungswesen“ und 45 aus dem Bereich „Bankbetriebslehre/Finanzierung“. Dabei sind acht Zeitschriften in beiden Bereichen vertreten, sodass insgesamt 72 Journals für die weitere Untersuchung herangezogen werden. Die Fokussierung auf die Bereiche „Rechnungswesen“ und „Bankbetriebslehre/ Finanzierung“ ist damit zu begründen, dass Conference Calls als Kommunikationsmittel am Kapitalmarkt für beide Fachrichtungen von Interesse sind. Mit der Auswahl von ausschließlich international anerkannten Zeitschriften werden die Qualität sowie die Rigorosität der Beiträge gewährleistet. ${ }^{5}$

Um relevante Beiträge in den ausgewählten Zeitschriften zu identifizieren, wurden internationale Fachzeitschriftendatenbanken (Web of Science, EBSCO, Science Direct sowie EconBiz) bis Ende 2018 durchsucht. Beiträge, die bis zum Ende des Untersuchungszeitraums (Ende 2018) akzeptiert wurden und im Laufe des Jahres

Tab. 2 Sample

Literaturauswahl für den Zeitraum 1998-2018

\begin{tabular}{lll}
\hline & Zeitschriften & Beiträge \\
\hline $\begin{array}{l}\text { Relevante Beiträge auf Basis der einschlägigen Suchbegriffe } \\
\text { im Titel und Abstract }\end{array}$ & 72 & 242 \\
$\begin{array}{l}\text { Abzüglich Beiträge, die Conference Calls nicht als Schwer- } \\
\text { punkt behandeln oder nicht ergebnisorientierte Conference }\end{array}$ & 52 & 182 \\
$\begin{array}{l}\text { Calls thematisieren } \\
\begin{array}{l}\text { Abzüglich Beiträge, die eine theoretische oder experimentel- } \\
\text { le Forschung darstellen }\end{array}\end{array}$ & 1 & 11 \\
\begin{tabular}{l} 
Für die Literaturanalyse relevante Papiere \\
\hline
\end{tabular} & 19 & 49 \\
\hline
\end{tabular}

Tab. 2 fasst die einzelnen Schritte im Selektionsprozess zusammen

\footnotetext{
4 Abrufbar unter http://vhbonline.org/service/jourqual/vhb-jourqual-3/. Abgerufen am 01.10.2019.

5 Um die Limitation der fehlenden Vollerhebung der Artikel abzumildern, haben wir die Suche um Beiträge aus Zeitschriften mit einem C/D Ranking erweitert. Die Suche ergab nur drei weitere relevante Beiträge.
} 
2019 gedruckt worden sind, werden ebenfalls in der Auswahl berücksichtigt. ${ }^{6}$ Die Suchbegriffe sind ,conference call“ sowie Begriffe aus angrenzenden Themenbereichen wie ,earnings related conference calls“, „,regulation FD“ und ,earnings call“. Durch diese Vorgehensweise ergaben sich 242 relevante Forschungspapiere. Die Durchsicht des Inhalts der Abstracts zeigte, dass sich 182 Studien schwerpunktmäBig nicht mit Conference Calls beschäftigen bzw. keine Conference Calls im Rahmen von Ergebnisberichterstattungen thematisieren. Zudem fokussiert sich unsere Literaturanalyse auf empirisch-quantitative Archivstudien, weswegen wir theoretische oder experimentelle Studien eliminieren. Nach diversen Bereinigungsschritten ergibt sich eine finale Auswahl von 49 Studien aus den Jahren 1998 bis 2018.

Tab. 2 fasst die einzelnen Schritte im Selektionsprozess zusammen. Tab. 3 gibt einen Überblick über die zeitliche Einordnung der Papiere sowie deren Klassifikation. Hierbei zeigt sich, dass im Jahr 2018 bisher die meisten Artikel zu Conference

Tab. 3 Sample

Ausgewählte Beiträge nach Jahr, Zeitschrift und Klassifikation (Die Einordnung erfolgt anhand des VHB-JOURQUAL3 Rankings.)

\begin{tabular}{|c|c|c|}
\hline Veröffentlichungsjahr & Zeitschrift & Klassifikation \\
\hline 2018: 11 & Abacus: 1 & $A+: 24$ \\
\hline 2017: 5 & Accounting Horizons: 1 & A: 11 \\
\hline 2016: 2 & Accounting, Organizations and Society: 1 & B: 14 \\
\hline 2015: 5 & Contemporary Accounting Research: 1 & \\
\hline 2014: 2 & European Accounting Review: 1 & \\
\hline 2013: 2 & European Financial Management: 1 & \\
\hline 2012: 6 & Financial Analysts Journal: 2 & \\
\hline 2011: 1 & Financial Management: 1 & \\
\hline 2010: 3 & Journal of Accounting and Economics: 4 & \\
\hline 2009: 0 & Journal of Accounting Research: 10 & \\
\hline 2008: 3 & Journal of Banking \& Finance: 1 & \\
\hline 2007: 0 & Journal of Behavioral Finance: 1 & \\
\hline 2006: 0 & Journal of Business Finance \& Accounting: 1 & \\
\hline 2005: 1 & Journal of Corporate Finance: 1 & \\
\hline 2004: 4 & Journal of Financial Markets: 1 & \\
\hline 2003: 1 & Review of Accounting Studies: 7 & \\
\hline 2002: 1 & Review of Quantitative Finance and Accounting: 4 & \\
\hline 2001: 0 & The Accounting Review: 9 & \\
\hline 2000: 0 & The Journal of Finance: 1 & \\
\hline \multicolumn{3}{|l|}{ 1999: 1} \\
\hline 1998: 1 & & \\
\hline
\end{tabular}

Tab. 3 gibt einen Überblick über die zeitliche Einordnung der Papiere sowie deren Klassifikation. Beiträge, die bis zum Ende des Untersuchungszeitraums (Ende 2018) akzeptiert wurden und im Laufe des Jahres 2019 gedruckt worden sind, werden ebenfalls in der Auswahl berücksichtigt

\footnotetext{
${ }^{6}$ Somit werden Brochet et al. (2019) und Heinrichs et al. (2019) im Sample zum Ende des Jahres 2018 berücksichtigt. Beiträge, welche bis zum Ende des Untersuchungszeitraums (Ende 2018) noch den Status eines Working Papers hatten, werden nicht explizit im Sample berücksichtigt. Diese werden jedoch ergänzend herangezogen, um neue Entwicklungen in der Literatur adäquat abzubilden.
} 
Calls publiziert wurden. Dies ist als Indiz dafür zu werten, dass es sich weiterhin um ein sehr aktives Forschungsfeld handelt.

\section{Analyse des Forschungsfelds Conference Calls}

Die systematische Analyse des Forschungsfelds Conference Calls erfolgt anhand eines Rahmenkonzepts. Dementsprechend werden die Studien in Beiträge zum Phänomen Conference Call als Offenlegungsinstrument, zu den Determinanten und den ökonomischen Konsequenzen von Conference Calls gruppiert (Abschn. 4.1, 4.2 und 4.3). Zunächst wird die Literatur analysiert, die das Phänomen Conference Call als Offenlegungsinstrument erforscht. Hierzu zählen Beiträge zum Informationsgehalt, der Darstellungsform sowie zur Nutzung von Conference Calls, ohne explizit auf die ökonomischen Wirkungen einzugehen. Die empirische Evidenz zu den Determinanten von Conference Calls identifiziert sowohl Faktoren, von denen die Durchführungsentscheidung als auch die Teilnahmemöglichkeit an einem Conference Call abhängen. Die dritte Kategorie in unserem Rahmenkonzept bilden Studien, welche die ökonomischen Konsequenzen von Conference Calls untersuchen. Das Hauptaugenmerk liegt auf ökonomischen Folgen in Form von Kapitalmarktreaktionen, Einflüssen auf Analystenprognosen sowie Auswirkungen auf Kapitalkosten. ${ }^{7}$ Ergänzend werden Studien vorgestellt, die Conference Calls als Informationsquelle für die Beantwortung anderer Forschungsfragen nutzen, d. h. in denen Conference Calls nicht der primäre Untersuchungsgegenstand sind (Abschn. 4.4). Vielmehr werden hier die Informationen aus Conference Calls beispielsweise dazu genutzt, Verhaltensweisen oder Charakteristika des Managements zu identifizieren. Abb. 1 fasst die Kategorien des Rahmenkonzepts unserer Literaturanalyse zusammen. Die Tab. 4-7 im Online Appendix gewähren einen inhaltlichen Überblick über die Studien der einzelnen Themenbereiche.

\subsection{Phänomen Conference Call als Offenlegungsinstrument}

\subsubsection{Informationsgehalt von Conference Calls}

Eine wesentliche Frage im Rahmen der Untersuchung von Conference Calls ist, ob diese zusätzliche, für den Kapitalmarkt relevante Informationen beinhalten. So untersuchen Studien einerseits, wovon deren Informationsgehalt abhängt. Andererseits diskutiert eine Vielzahl von Beiträgen deren Verhältnis gegenüber anderen Offenlegungskanälen. Gerade der Q\&A-Teil eines Conference Calls ermöglicht es Analysten, aktiv Informationen nachzufragen und damit Einfluss auf die veröffentlichten Inhalte zu nehmen. Bischof et al. (2014) untersuchen die Verwendung von Fair Value-bezogenen Informationen im Rahmen des Entscheidungsprozesses von Ana-

\footnotetext{
7 Im Rahmen der thematischen Kategorisierung der Beiträge wird stets auf die Haupterkenntnis der Papiere abgestellt und auf Doppelzuordnungen verzichtet. Die einzige Ausnahme bilden die Studien von Frankel et al. (1999) und Bushee et al. (2003), die jeweils explizit sowohl Determinanten als auch ökonomische Wirkungen von Conference Calls untersuchen. Diese werden entsprechend in beiden Abschnitten erfasst.
} 


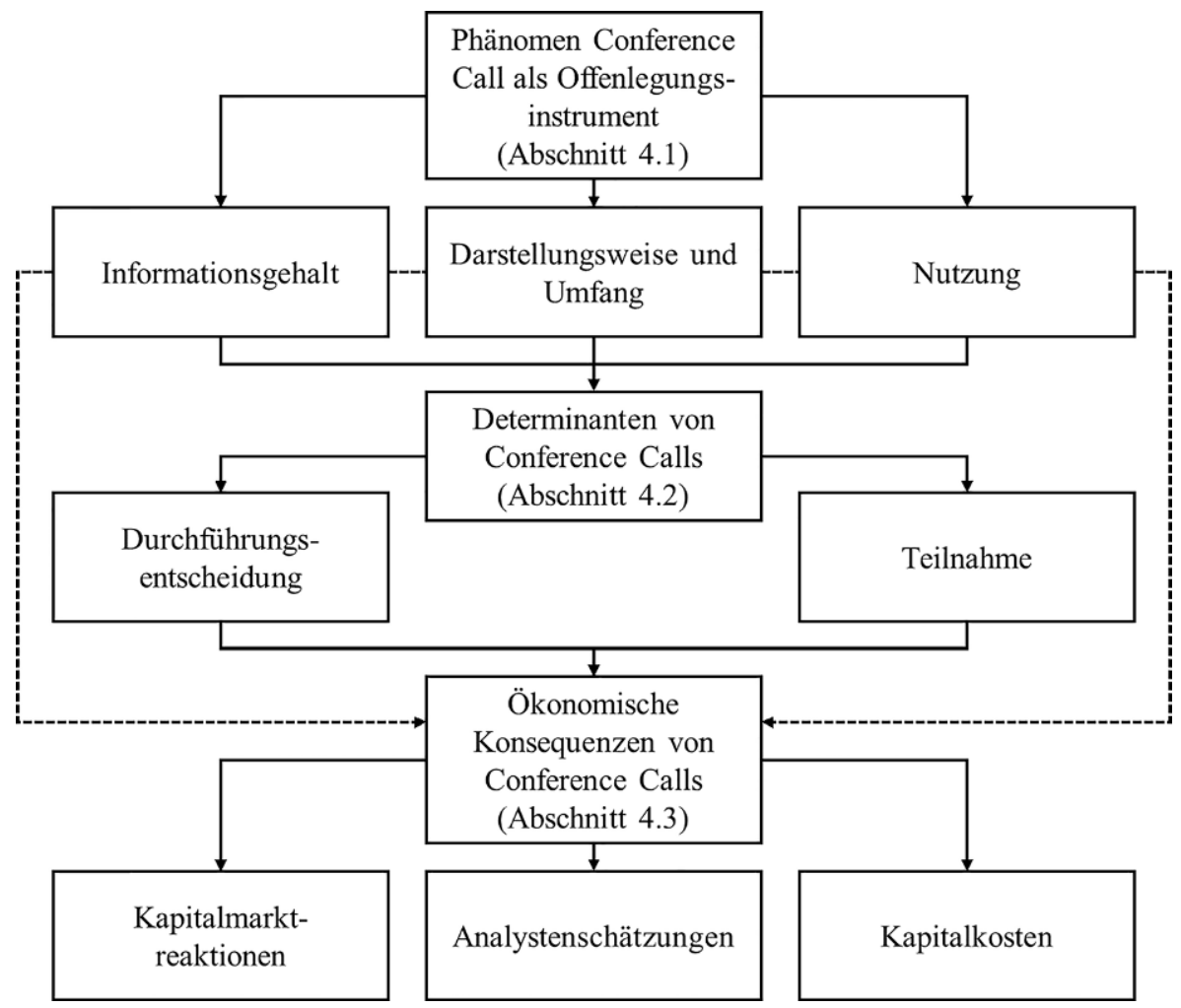

Abb. 1 Rahmenkonzept zur Strukturierung der Artikel zu Conference Calls

lysten bei Banken. Die Untersuchung zeigt, dass insbesondere die Reklassifizierung von finanziellen Vermögenswerten und die Fair Value-Option bei Verbindlichkeiten von Interesse sind. Grundsätzlich steigt die Wahrscheinlichkeit einer Fair Valuebezogenen Frage mit der zunehmenden Bedeutung der Fair Value-Bilanzierung für den Abschluss. Die Autoren folgern, dass die unterlassene oder reduzierte Berichterstattung im Finanzbericht bei Vermögenswerten zu mehr themenspezifischen Fragen führt. Demnach nehmen Conference Calls eine substituierende Rolle ein. AnschlieBend untersuchen Bischof et al. (2014) Analystenreports auf Fair Value-bezogene Informationen. Dabei bestätigt sich die Annahme, dass auch hier die Wahrscheinlichkeit einer Aussage zu Fair Values mit der Bedeutung für den Abschluss steigt.

Der Diskussionsteil eines Conference Calls bietet die Möglichkeit, Aussagen des Managements aus dem Präsentationsteil zu hinterfragen oder Themen zu adressieren, die bisher nicht durch das Management selbst angesprochen wurden. Hollander et al. (2010) untersuchen in ihrer Studie, ob das Management durch das Nichtbeantworten von Fragen Informationen zurückhält und wie dieses Verhalten vom Kapitalmarkt interpretiert wird. Die manuelle Auswertung von Protokollen ergibt, dass in etwa $60 \%$ der Conference Calls zumindest eine Frage nicht beantwortet wird. Mit einem Anteil von 59\% der nicht beantworteten Fragen dominiert thematisch der Bereich Finanzen, gefolgt von Fragen zum Geschäftsbetrieb mit $31 \%$. In Bezug auf den 
Zeithorizont werden mit $74 \%$ die meisten Fragen zu aktuellen Themen nicht beantwortet. Das Zurückhalten von Informationen durch das Management deutet nach Ansicht von Hollander et al. (2010) auf hohe Offenlegungskosten hin und wird am Kapitalmarkt als negative Information aufgefasst.

Eine weitere denkbare Möglichkeit spezifische Informationen nicht preiszugeben ist eine schwer verständliche Beantwortung der Analystenfragen. In diesem Zusammenhang zeigt die Studie von Bushee et al. (2018) den Einfluss der Komplexität der Kommunikation auf den Informationsgehalt eines Conference Calls. Die Messung von Komplexität mit einfachen Maßen aus der Linguistik wie beispielsweise dem FOG Index ist in der Forschung umstritten (Loughran und McDonald 2016). Ein hoher FOG Indexwert könnte dabei einerseits für einen komplexen Text stehen, welcher schwer lesbar ist und damit den Informationsgehalt mindert bzw. die Informationsverarbeitung durch Analysten und Investoren erschwert. Andererseits kann ein hoher Wert auch als Maß für den Informationsgehalt eines Textes verstanden werden (Kaya und Seebeck 2018), wenn beispielsweise neue Details zu technischen Innovationen kommuniziert werden. Bushee et al. (2018) begegnen diesem Spannungsfeld mit der Aufteilung der Komplexität in einen Teil, der für die Erläuterungen von komplexen Sachverhalten nötig ist und somit zum Verständnis beiträgt (latent information), und einen Teil, der zur Verschleierung von Informationen führt (obfuscation). Die Sprache der Analysten ist dabei annahmegemäß frei von verschleiernder Komplexität, wohingegen das Management beide Komponenten der Komplexität verwendet. Somit dienen die Analysten als Benchmark für die „nötige“ Komplexität. Die Ergebnisse zeigen, dass der Teil der Komplexität zur Verschleierung (Beschreibung komplexer Sachverhalte) positiv (negativ) mit den Informationsasymmetrien zwischen Unternehmen und Kapitalmarktteilnehmern zusammenhängt.

Im Rahmen der Ergebnisveröffentlichung nutzen Unternehmen neben Conference Calls auch Pressemitteilungen. Dabei stellt sich die Frage, inwieweit der Präsentations- bzw. Diskussionsteil Zusatzinformationen gegenüber Pressemitteilungen beinhaltet und ob deren Informationsgehalt von der Unternehmensleistung abhängt. Die Ergebnisse von Matsumoto et al. (2011) weisen darauf hin, dass beide Teile für den Markt zusätzlich informativ sind. Dabei weist der Diskussionsteil aufgrund der freieren Kommunikation einen höheren Informationsgehalt auf als der Präsentationsteil. Hinsichtlich der Unternehmensleistung stellen Matsumoto et al. (2011) fest, dass Manager bei schlechter Performance nicht ausreichend Informationen im Präsentationsteil veröffentlichen. Ein Teil der fehlenden Informationen wird allerdings im Gegenzug durch Fragen im Diskussionsteil publik. Außerdem deutet die Häufigkeit von finanziellen und zukunftsbezogenen Wörtern in Conference Calls darauf hin, dass Manager bei schlechter Performance dazu tendieren, im Präsentationsteil mehr über zukünftige Ereignisse zu berichten und bei guter Unternehmensleistung eher auf finanzielle Inhalte eingehen (Matsumoto et al. 2011). Die Identifikation des inkrementellen Anteils neuer Informationen aus Conference Calls kann auch durch moderne Methoden der computergestützten Textanalyse gemessen werden. So ermittelt Cicon (2017) mit Hilfe von computergestützter Textanalyse die Ähnlichkeit (similarity) zwischen beiden Teilen des Conference Calls und findet einen höheren Informationsgehalt des Diskussionsteils bei geringerer inhaltlicher Über- 
schneidung mit dem Präsentationsteil. Insgesamt legen sowohl die Ergebnisse der Studie von Matsumoto et al. (2011) als auch die von Cicon (2017) eine Steigerung des Informationsnutzens durch die Mitwirkung von Analysten nahe. Demgegenüber verliert der Conference Call wertrelevante Informationen, wenn der Q\&A-Teil vom Management dominiert wird (Cicon 2017).

Offene Forschungsfragen Bislang wurden in der Fachliteratur nur wenige Studien veröffentlicht, die sich detailliert mit den in Conference Calls kommunizierten Inhalten befassen. Neben der Studie von Hollander et al. (2010) stammt die einzige Studie, die sich mit den spezifischen Inhalten von Conference Calls auseinandersetzt von Tasker (1998b). In dieser Studie untersucht die Autorin manuell für 12 Conference Calls die Themengebiete der von Analysten gestellten Fragen. Tiefergehende Inhaltsanalysen für eine Vielzahl von Conference Call Protokollen könnten neue wertvolle Erkenntnisse liefern. Die Analyse der behandelten Themen könnte zum einen Aufschluss darüber geben, welche Themen Manager freiwillig veröffentlichen bzw. näher erläutern und andererseits, welche Themen für Teilnehmer von besonderem Interesse sind. Insbesondere Erkenntnisse über den Informationsbedarf von Analysten können Unternehmen helfen, die Inhalte ihrer Berichterstattung besser an die Bedürfnisse des Kapitalmarkts anzupassen. Eine genauere Identifikation der Informationsbedürfnisse von Kapitalmarktteilnehmern kann auch für Standardsetter, wie das IASB oder das FASB, hilfreich sein. So könnten die Erkenntnisse, insbesondere im aktuellen Kontext der Informationsüberflutungsdebatte, bei der zielgerichteten Weiterentwicklung bestehender Standards der regulierten Berichterstattung helfen. ${ }^{8}$

Zudem gibt es noch wenige Erkenntnisse darüber, ob Conference Calls eine substituierende oder ergänzende Rolle gegenüber der traditionellen Finanzberichterstattung zukommt. So untersuchen Bischof et al. (2014) Conference Calls und Finanzberichte von Banken und sehen in Conference Calls eher eine substituierende Rolle, während Bassemir et al. (2013) diesen eine ergänzende Rolle zusprechen. Völlig offen in der Literatur ist auch das Zusammenwirken von verschiedenen Kommunikationskanälen wie Conference Calls und neuen Medien (wie beispielsweise soziale Netzwerke). Denkbar ist in diesem Zusammenhang, dass Unternehmen ihre Kommunikationsstrategie auf die jeweiligen Adressaten abstimmen. So könnten beispielsweise Erläuterungen zu komplexen Themen eher über Conference Calls als über soziale Medien veröffentlicht werden.

\subsubsection{Darstellungsweise und Umfang von Conference Calls}

Auch die Aspekte des Umfangs bzw. der Darstellungsweise von Informationen innerhalb von Conference Calls werden von verschiedenen Studien empirisch untersucht.

\footnotetext{
8 Neben den Initiativen des IASB (IASB 2017) gibt es weitere internationale Bestrebungen den Informationsgehalt und die Nützlichkeit der qualitativen Berichterstattung zu verbessern. So hat beispielsweise das FASB um Stellungnahmen zur Bewertung der aktuellen textuellen Berichterstattung gebeten (FASB 2012). Auch die SEC führt seit dem Jahr 2013 ihr sogenanntes „Disclosure effectiveness“-Projekt (SEC 2013) durch, um die Effizienz der Berichterstattung zu überprüfen.
} 
So befassen sich Frankel et al. (2010) in ihrer Arbeit mit der Frage, ob das Verfehlen einer Ergebnisprognose um einen Cent größere Auswirkungen auf den Umfang der Berichterstattung hat als höhere Abweichungen. In der Literatur wird angenommen, dass Manager es möglichst vermeiden, Ergebnisprognosen knapp zu verfehlen, da sie glauben, dass der Kapitalmarkt diese besonders hart bestraft (sog. ,miss-by-thepenny-effect“) (Graham et al. 2005). Zudem benötigen Manager mehr Zeit für die Erläuterung knapper Abweichungen und haben daher weniger Möglichkeiten auf zukünftige Entwicklungen einzugehen (Graham et al. 2005). Die Arbeit zeigt, dass die Länge von Conference Calls bei einer knappen Verfehlung der Prognose stärker steigt als in den angrenzenden Intervallen mit höheren Abweichungen. Darin sehen die Autoren ein Indiz für einen erhöhten Informationsbedarf, der sich durch das Ausmaß der Nachfragen zeigt.

Conference Call Transkripte bieten für Forscher eine gute Möglichkeit, die Kommunikationsweise im Detail zu analysieren. So können Davis et al. (2015) mit Hilfe von Textanalyse zeigen, dass das Sentiment in Conference Calls signifikant mit dem Optimismus und anderen Charakteristika der teilnehmenden Manager zusammenhängt. Mit der ethnischen Herkunft des Managements identifizieren Brochet et al. (2019) in ihrer Studie einen weiteren möglichen Einflussfaktor auf das Kommunikationsverhalten. Die sprachlichen Einflüsse finden sich dabei eher im interaktiven Q\&A-Teil und weniger im bereits im Vorfeld verfassten Präsentationsteil wieder. In ähnlicher Weise untersuchen Levy et al. (2018) den Zusammenhang zwischen der Kommunikationsstrategie und der persönlichen Haftung von CFOs. Sie finden im Falle von persönlicher Haftung neben einer eher konservativen Rechnungslegungsund Offenlegungspolitik einen negativen Effekt auf den textuellen Ton in der Conference Call Kommunikation. Die Schlussfolgerung der Studie ist, dass CFOs bei persönlicher Haftung sensibler reagieren und entsprechend konservativer berichten als bei bloßer Haftung der Gesellschaft.

Offene Forschungsfragen Da Conference Calls inzwischen teilweise per Videokonferenz übertragen werden, ermöglicht die Untersuchung dieses Kommunikationsmediums einen weiteren Ansatz für zukünftige Studien. Durch Videoanalysen können beispielsweise der Gesichtsausdruck, die Körperhaltung, Gesten oder das äußere Erscheinungsbild (z.B. Krawattenfarbe) untersucht werden, die in Texten oder Audiodateien nicht auswertbar sind (Mayew und Venkatachalam 2012; Price et al. 2012). Dies ermöglicht im Zusammenspiel mit anderen Forschungsdisziplinen, etwa der Psychologie oder den Kommunikationswissenschaften, eine vielschichtige Auswertung und könnte wichtige Hinweise zur Wirkung sowie zum Stellenwert der nonverbalen Kommunikation liefern. Die Analyse der nonverbalen Kommunikation ist insbesondere zwischen Management und Analysten (Larcker und Zakolyukina 2012; Price et al. 2012) sowie innerhalb eines Managementteams von Interesse (Price et al. 2012). Zwischen Management und den Analysten könnte untersucht werden, ob die nonverbale Kommunikation der Analysten Auswirkungen auf die Art und Weise, den Umfang oder die grundsätzliche Entscheidung zur Beantwortung der Fragen hat. Die nonverbale Kommunikation innerhalb des Managementteams könnte hingegen Aufschluss über die Rolle der einzelnen Manager geben, die möglicherweise auch deren Kommunikationsweise beeinflusst. 


\subsubsection{Nutzung von Conference Calls}

Eine wichtige Frage stellt die Identifikation der eigentlichen Informationsempfänger von Conference Calls dar. Die Studie von Heinrichs et al. (2019) zeigt mit Hilfe von Aufrufzahlen von Audio- bzw. Textprotokollen auf Thomson Reuters StreetEvents, dass neben den typischen Adressaten wie Analysten und Investoren auch andere Stakeholder wie Lieferanten, Banken oder Wettbewerber die Informationen aus Conference Calls nutzen. Innerhalb der Gruppe der institutionellen Investoren findet die Studie eine intensivere Nutzung durch am Unternehmen beteiligte Investoren gegenüber nicht beteiligten Investoren. Die Studie legt zudem nahe, dass die Nachfrage nach Informationen aus Conference Calls insbesondere von firmenspezifischen Faktoren (z. B. Verschuldung) abhängt.

Offene Forschungsfragen Die Weiterentwicklung des Forschungsfelds zur Nutzung von Conference Calls lässt weitere Untersuchungen offener Forschungsfragen zu. So zeigen beispielsweise Heinrichs et al. (2019), dass auch Wettbewerber an den Informationen aus Conference Calls interessiert sind. So könnte ein möglicher Grund gegen eine offene Ausgestaltung von Conference Calls in Wettbewerbskosten liegen, die einem Unternehmen entstehen können, wenn es wettbewerbssensible Informationen preisgibt. Ähnliche Auswirkungen könnten Wettbewerbskosten auch auf die Preisgabe von spezifischen Informationen zu Produkten, Margen oder Strategien innerhalb des Diskussionsteils haben. Überwiegen die Kosten der Preisgabe von Informationen den Nutzen, so wird sich das Management gegen die Offenlegung im Rahmen von Conference Calls entscheiden. So weigert sich beispielsweise der CEO von Apple, Tim Cook, konkrete Aussagen zu den Margen und Umsätzen der Apple Watch zu tätigen und verweist stattdessen auf mögliche Wettbewerbsnachteile bei Preisgabe dieser Informationen. Die bisherige Literatur zu den wettbewerbsbedingten Offenlegungskosten konzentriert sich fast ausschließlich auf die Veröffentlichung von Jahresabschlüssen (Dedman und Lennox 2009; Bernard et al. 2018a) oder deren Bestandteilen wie die Segmentberichterstattung (Botosan und Stanford 2005). Gegenüber diesen Studien bietet die Untersuchung von Wettbewerbskosten auf Basis von Conference Calls mehrere Vorteile. Während beispielsweise gewisse Informationen aus idiosynkratischen Gründen (nicht) im Jahresabschluss vorhanden sein können (z.B. Unklarheit über Offenlegungswahlrechte), bietet eine Nichtbeantwortung von Fragen im Diskussionsteil eine klarere Identifikation der bewussten Zurückhaltung von Informationen. Des Weiteren gewähren Conference Calls durch die Auswertung auf Ebene von individuellen Fragen detailliertere Einblicke in verschiedene wettbewerbssensible Themenbereiche. Insgesamt scheint daher eine Analyse des Zusammenhangs zwischen Wettbewerbskosten und Conference Calls ein interessantes Forschungsvorhaben zu sein. 


\subsection{Determinanten von Conference Calls}

\subsubsection{Durchführungsentscheidung}

Die ersten US-amerikanischen Studien im Forschungsfeld Conference Calls untersuchen die Determinanten der Durchführungsentscheidung, welche in Abwesenheit einer gesetzlichen Regulierung im Ermessen des Managements liegt. ${ }^{9}$ Eine mögliche theoretische Erklärung für die freiwillige Berichterstattung liegt in der geringen Aussagekraft der Finanzberichterstattung (Verrecchia 1990). Reicht die verpflichtende Berichterstattung nicht aus, das Unternehmen, dessen Geschäftsmodell oder dessen Entwicklung darzustellen, können freiwillige Veröffentlichungen helfen, diese Informationslücke zu schließen. Vor diesem Hintergrund deuten die Ergebnisse der Studie von Tasker (1998a) darauf hin, dass der Informationsgehalt der Finanzberichterstattung in einem negativen Zusammenhang zur Wahrscheinlichkeit des Ausrichtens eines Conference Calls steht. Somit können Unternehmen Informationskanäle außerhalb der verpflichtenden Finanzberichterstattung nutzen, um die Bedürfnisse des Kapitalmarkts zu bedienen.

Conference Calls werden zudem häufig von Unternehmen genutzt, die im Durchschnitt größer sind, denen mehr Analysten folgen und die öfter Eigen- oder Fremdkapital ausgeben. Darüber hinaus stammen die Unternehmen häufig aus Hightech Branchen, haben höhere Wachstumsraten und weisen ein überdurchschnittliches MarktBuchwert-Verhältnis auf (Frankel et al. 1999). Dies bestätigen die Erkenntnisse aus anderen Arbeiten zur freiwilligen Berichterstattung, wie etwa Lang und Lundholm (1993). Die Entscheidung Conference Calls auszurichten kann auch durch institutionelle Investoren beeinflusst werden. Für das Schwellenland Taiwan legt die Studie von Liang et al. (2012) nahe, dass die Beteiligung von ausländischen institutionellen Investoren die Anzahl der Conference Calls erhöht.

Einen weiteren möglichen Einfluss - durch sogenannte Netzwerkeffekte - finden Chan et al. (2017) in der Untersuchung von Conference Calls in Taiwan. Netzwerkeffekte implizieren zumeist eine gegenseitige Beeinflussung von zwei Objekten. Im Unternehmenskontext können Netzwerkeffekte entstehen, wenn Manager in Boards von unterschiedlichen Unternehmen sitzen. Die Ergebnisse der Autoren deuten darauf hin, dass Netzwerkeffekte zu einer höheren Durchführungswahrscheinlichkeit und Frequenz von Conference Calls beitragen. Als einen möglichen Erklärungsansatz führen die Autoren an, dass sich durch eine doppelte Managementrolle Offenlegungspraktiken und Unternehmensinformationen zu Märkten und Produkten, insbesondere in forschungsintensiven Hightech Unternehmen, schneller verbreiten.

Offene Forschungsfragen Zusammenfassend werden Fragen zur grundsätzlichen Durchführungsentscheidung vielseitig untersucht. Bislang liefert jedoch keine Studie tiefergehende Erkenntnisse zur strategischen Planung und Durchführung von Conference Calls. So bleibt beispielsweise die Frage der Festsetzung des Zeitpunkts der

\footnotetext{
9 Gegenüber Deutschland existiert in den anderen hier untersuchten Rechtsräumen keine Pflicht zur Durchführung. Insofern ist keine direkte Übertragung der Ergebnisse zur Durchführungsentscheidung auf andere Rechtsräume mit abweichenden Vorschriften möglich.
} 
Ausrichtung ungeklärt, d.h. ob Unternehmen bewusst abwarten, bis Peer-Unternehmen (wie beispielsweise Konkurrenten) zunächst berichten oder den Conference Call strategisch vor denen der Peer-Unternehmen ausrichten. Vorgelagert zu dieser Entscheidung ist natürlich die Festsetzung des Veröffentlichungsdatums von Quartalsund Jahresabschlüssen. Hier zeigen Johnson und So (2018), dass Unternehmen, die später veröffentlichen als ursprünglich erwartet, schlechtere Nachrichten berichten als jene, die das ursprüngliche Veröffentlichungsdatum vorziehen. Somit haben Unternehmen durchaus Flexibilität hinsichtlich des konkreten Offenlegungszeitpunkts. Ferner zeigt die bisherige Offenlegungsliteratur, dass sich kleinere Unternehmen an größeren Unternehmen derselben Branche orientieren (Leary und Roberts 2014; Bernard et al. 2018b). Insgesamt erscheint eine Analyse der strategischen Planung von Conference Calls eine vielversprechende Forschungsrichtung für die Zukunft.

\subsubsection{Teilnahme an Conference Calls}

Eine Reihe von Studien untersucht, wer direkten Zugang zu Conference Calls als Informationsquelle erhält und in welchem Umfang die Teilnahmemöglichkeit ausgeübt wird. Da es US-Unternehmen vor Reg FD grundsätzlich freistand, den Zugang zu ihren Conference Calls auf bestimmte Gruppen zu beschränken oder für alle Marktteilnehmer zu öffnen, untersuchen Bushee et al. (2003), von welchen Faktoren die Entscheidung des Managements abhängt. Die Studie zeigt, dass Unternehmen mit einer größeren Zahl an Aktionären, einem geringeren Anteil institutioneller Anleger sowie Unternehmen, denen weniger Analysten folgen, eher offene Conference Calls ausrichten. Außerdem veranstalten Unternehmen mit einem größeren Anteil immaterieller Vermögenswerte seltener offene Conference Calls. Dies lässt vermuten, dass Unternehmen komplexe Sachverhalte bevorzugt mit fachkundigen Teilnehmern austauschen (Bushee et al. 2003).

Unabhängig vom generellen Zugang kann es auch innerhalb von Conference Calls zu einer Ungleichbehandlung der Teilnehmer kommen. Fragende können gegenüber nicht fragenden Analysten Informationsvorteile erzielen, da die während des Conference Calls veröffentlichten Informationen die privaten Informationen der einzelnen Teilnehmer gezielt erweitern können. Mayew (2008) zeigt, dass die Wahrscheinlichkeit einer Fragemöglichkeit eines Analysten steigt, wenn der Analyst das Unternehmen im Rahmen einer Aktienempfehlung vor dem Conference Call positiv bewertet. Angesehene Analysten werden dabei für positive (negative) Beurteilungen stärker belohnt (geringer bestraft) als weniger angesehene Analysten.

Ein Großteil der bisherigen Studien untersucht die generellen Teilnahmemöglichkeiten, ohne dabei differenziert auf verschiedene Gruppen von Teilnehmern und deren mögliche Motive einzugehen. Jung et al. (2018) sind die ersten, die explizit buy-side Analysten als Teilnehmer von Conference Calls analysieren. Die Autoren zeigen, dass die Wahrscheinlichkeit von buy-side Analysten an Conference Calls zu partizipieren negativ mit den öffentlich verfügbaren Informationen des jeweiligen Unternehmens zusammenhängt. In einem aktuellen Arbeitspapier liefern Call et al. (2018) weitere Erkenntnisse über deren Teilnahme. So nehmen buy-side Analysten, die bei einem Hedgefonds oder einem registrierten Anlageberater tätig sind, eher an einem Conference Call teil als jene, die für einen Investmentfonds arbeiten. Die Au- 
toren finden außerdem Indizien dafür, dass das Management zwischen Fragen von buy-side und sell-side Analysten diskriminiert und die Kommunikation mit ersteren vergleichsweise kurz und weniger positiv ist.

Sowohl die Studien zur Durchführungsentscheidung als auch zum Zugang zu Conference Calls stehen vor dem Grundproblem der simultanen Kausalität. Diese entsteht, wenn entgegen der postulierten Wirkungsweise die abhängige Variable gleichzeitig auf eine oder mehrere unabhängige Variablen wirkt. Die Studie von Bushee et al. (2003) findet beispielsweise einen Einfluss von Unternehmenscharakteristika (unabhängige Variablen) auf die Entscheidung eines Unternehmens, Conference Calls offen zu gestalten (abhängige Variable). Jedoch könnten die Ergebnisse der Studie auch so interpretiert werden, dass eine offene Durchführung von Conference Calls die Charakteristika des Unternehmens (wie etwa die Anzahl der folgenden Analysten) beeinflusst. Ein Teil der Studien begegnet diesem Problem beispielsweise mit Instrumentvariablenschätzungen (Liang et al. 2012). Andere validieren ihre Ergebnisse durch Schätzungen von Lag-Modellen (Bushee et al. 2003) oder detaillierten Querschnittsuntersuchungen.

Offene Forschungsfragen Die bisherige Literatur untersucht bereits eine Vielzahl von Aspekten zur Teilnahme an Conference Calls. Neben Conference Calls können Analysten allerdings weitere Quellen der (privaten) Informationsbeschaffung wie beispielsweise persönliche Gespräche mit dem Management nutzen. So zeigen Cheng et al. (2016), dass Analysten, die im häufigeren Kontakt mit Unternehmensvertretern stehen, eine höhere Prognosegenauigkeit haben. Daher ist es denkbar, dass das Einräumen von persönlichen Treffen eine wichtige Rolle bei der Teilnahmeentscheidung spielt. Entsprechend könnten Analysten, denen die Möglichkeit eines persönlichen Treffens mit dem Management eingeräumt wird, weniger an der Teilnahme oder zumindest einer aktiven Beteiligung an einem Conference Call interessiert sein. Als Folge sind die Ergebnisse der bisherigen Studien mit größerer Vorsicht zu interpretieren. Die Identifikation von privaten Treffen mit dem Management stellt eine Herausforderung dar, da Unternehmen in der Regel gar nicht oder nur unzureichend öffentlich über solche informieren. Einen möglichen Lösungsansatz hierzu bietet die Untersuchung von Unternehmen der Shenzhen Stock Exchange (SZSE). Diese sind gemäß den SZSE Information Fair Disclosure Guidelines da$\mathrm{zu}$ verpflichtet, eine Übersicht $\mathrm{zu}$ veröffentlichen, die wesentliche Informationen über private Treffen mit Analysten beinhaltet (Cheng et al. 2016; Bowen et al. 2018). Insgesamt stellt die Untersuchung der Interaktion zwischen Conference Calls und anderen Möglichkeiten der (privaten) Informationsbeschaffung eine interessante Forschungsfrage dar.

\section{3 Ökonomische Konsequenzen von Conference Calls}

\subsubsection{Kapitalmarktreaktionen}

Bereits die ersten empirischen Untersuchungen befassen sich mit den Auswirkungen von Conference Calls auf den Kapitalmarkt. Frankel et al. (1999) analysieren die Volatilität der Aktienrendite und das Handelsvolumen während Conference Calls. 
Die Untersuchung weist auf eine erhöhte Volatilität der Aktienkursrendite während Conference Calls im Vergleich zu Kontrollperioden ohne Conference Calls hin. Ebenso lassen sich erhöhte Handelsvolumina feststellen (Frankel et al. 1999). Im Gegensatz dazu finden Dell' Acqua et al. (2010) mit Fokus auf die Hightech Industrie eine sinkende Volatilität des Aktienkurses bei einem vermehrten Einsatz von Conference Calls. ${ }^{10}$ Zudem legt die Analyse der durchschnittlichen Handelsvolumina nahe, dass insbesondere große institutionelle Investoren im Laufe von Conference Calls handeln und somit Informationen sofort verwerten. Demgegenüber verfügen kleine Investoren ohne Zugang zu Conference Calls nicht über die gleichen Informationen (Frankel et al. 1999).

Nicht alle am Kapitalmarkt veröffentlichten Informationen werden sofort, sondern über einen längeren Zeitraum hinweg von den Marktteilnehmern verarbeitet. Dieses Phänomen wird als ,post-earnings-announcement drift“ bezeichnet (Bernard und Thomas 1989). Kimbrough (2005) zeigt, dass der post-earnings-announcement drift und der Anteil der Gesamtmarktreaktionen, der sich auf den nachgelagerten Teil der Ergebnisbekanntgabe bezieht, beim Einsatz von Conference Calls vermindert wird. Trotz gesteigertem Informationsumfang durch Conference Calls scheinen Investoren die größere Menge an Informationen zur Beurteilung jedoch nicht zu nutzen.

Seit der Einführung der Reg FD in den USA, sind Unternehmen unter anderem dazu verpflichtet, Conference Calls für alle Interessierten zu öffnen und damit den gesamten Kapitalmarkt gleichermaßen mit Informationen zu versorgen. Für Wissenschaftler ist dies eine gute Gelegenheit, die Auswirkung der Gesetzesänderung auf die Unternehmen und den Kapitalmarkt zu beobachten. So ist die Frage, ob Investoren unterschiedlich auf offene und geschlossene Conference Calls reagieren, Gegenstand von diversen Untersuchungen. Da Informationen durch eine offene Ausgestaltung einem größeren Investorenkreis zugänglich sind, vermuten Bushee et al. (2003) eine Erhöhung der Preisvolatilität und des Handelsvolumens durch die unterschiedliche Bewertung von institutionellen und privaten Investoren. Die Studie findet zwar eine höhere Aktienkursvolatilität für offene Conference Calls, aber kein höheres Handelsvolumen, was auf eine ähnliche Einschätzung der Informationen durch die Marktteilnehmer hindeutet.

Der veränderte Informationszugang durch die Öffnung von Conference Calls wirkt sich auch auf die Informationsmöglichkeiten diverser Kapitalmarktteilnehmer aus. Aufgrund der Erweiterung des Informationszugangs auf eine breitere Investorengruppe, wird bei Unternehmen, die zuvor geschlossene Conference Calls ausgerichtet haben, die Preisvolatilität vermutlich stärker steigen als bei Unternehmen, die diese bereits vor der Gesetzesänderung offen ausgerichtet haben. Bushee et al. (2004) finden diesen Effekt, Lee et al. (2004) jedoch nicht. Die Analyse der Handelstransaktionen während Conference Calls zeigt eine erhöhte Aktivität von kleineren Investoren durch die Einführung der Reg FD (Bushee et al. 2004; Lee et al. 2004). Zudem untersuchen Lee et al. (2004) mit Hilfe des bid-ask spread, ob sich die Neu-

\footnotetext{
10 Der Unterschied der Ergebnisse der oben genannten Studien hinsichtlich der Volatilität könnte neben der Branchenspezifität insbesondere durch die unterschiedlichen Betrachtungszeiträume bei der Berechnung der Volatilität begründet sein. Während Frankel et al. (1999) Volatilitäten auf Basis von Intraday-Daten berechnen, berechnen Dell'Acqua et al. (2010) die Volatilität auf jährlicher Basis.
} 
regelung auf die Handelskosten der Investoren auswirkt. Sie vermuten, dass sich bei gleichem Informationszugang für alle Marktteilnehmer die Komponente der adversen Selektion im bid-ask spread reduziert. Einen gegenläufigen Effekt erwarten die Autoren, wenn Unternehmen durch die Reg FD weniger Informationen veröffentlichen. Die Studie findet keine signifikante Veränderung der Komponente der adversen Selektion durch die gesetzliche Änderung, sodass das erhöhte Informationsvolumen vermutlich keine Auswirkung auf die Informationsasymmetrien hat (Lee et al. 2004).

Zudem beeinflusst die Regulierung auch institutionelle Investoren. Ke et al. (2008) untersuchen das Handelsverhalten von institutionellen Investoren, die auf kurzfristige Gewinne spekulieren. Im Zeitraum vor der Änderung kann ein erhöhtes Verkaufsverhalten von institutionellen Investoren festgestellt werden (Ke und Petroni 2004), welches die Autoren zumindest teilweise auf den Zugang zu privaten Informationen zurückführen. Ein solches Verhalten ist nach Einführung der Gesetzesänderung nicht mehr zu beobachten (Ke et al. 2008).

Sowohl Untersuchungen vor als auch nach Reg FD gehen mit ökonometrischen Problemen einher. So weisen Studien vor Reg FD das Problem der endogenen Selbstselektion auf. Der analysierte Datensatz beruht dabei auf einer Stichprobe, die nicht nach dem Zufallsprinzip erhoben wird. Studien, welche die Ausrichtung von offenen versus geschlossenen Conference Calls untersuchen, stehen vor der Herausforderung, dass herkömmliche Regressionsmethoden zu inkonsistenten und verzerrten Schätzern führen können. ${ }^{11}$ Die Sample-Selection Korrektur wird daher häufig durch das Heckman-Zweistufen-Verfahren vorgenommen (Bushee et al. 2003; Jung et al. 2018). Studien nach Einführung der Reg FD stehen vor dem Grundproblem, dass es sich bei der Regulierung nicht um einen exogenen Schock handelt, sondern der Gesetzgeber vielmehr die vorherige Benachteiligung von Kapitalmarktteilnehmern bewusst durch die Gesetzesänderung einschränken möchte. ${ }^{12}$

Manager verfügen über private Informationen und die Gründe für das Vorenthalten von Informationen bzw. die Verzerrung der Informationslage können vielfältig sein. Eine mögliche Folge der Zurückhaltung von Informationen ist die kognitive Dissonanz. Diese beschreibt einen Zustand, bei dem das Gefühl und Wissen des Managers auf Basis privater Informationen nicht zur getroffenen Aussage passt und somit ein innerliches Spannungsverhältnis erzeugt. Liegt dadurch bei Managern eine negative emotionale Stimmungslage (wie beispielsweise Angst oder Verärgerung) vor, so nehmen Mayew und Venkatachalam (2012) an, dass diese über negative Informationen verfügen, die auf schlechte Nachrichten oder Unsicherheiten bezüglich guter Nachrichten hinweisen. Mittels LVA-Software (Layered Voice Analysis) bestimmen sie das emotionale und kognitive Level des Sprechenden in Conference Calls. Nach Berechnung eines quantitativen Werts für das Level von kognitiver Dissonanz, ordnen die Autoren den Managern ab einem bestimmten Wert eine negative emotionale Stimmungslage zu. Ähnlich verhält es sich mit dem emotionalen Level,

\footnotetext{
11 In ähnlicher Weise kann sich diese Problematik auch aus der generellen Entscheidung zur Durchführung von Conference Calls ergeben, welche entsprechend auch bei Studien nach Reg FD auftreten kann.

12 Die Studien begegnen diesem Problem u. a. mit Differenzen-von-Differenzen-Tests (z. B. Kross und Suk 2012). Dagegen gehört der Einsatz von Instrumentenvariablen zur Ausnahme (Liang et al. 2012).
} 
welches durch die Begeisterung des Managers gemessen wird und anhand dessen man auf eine positive Stimmung schließen kann. Eine positive emotionale Stimmungslage lässt auf gute Nachrichten über die zukünftige Unternehmensentwicklung schließen. Demgegenüber deutet eine negative Stimmungslage auf schlechte Nachrichten hin (Mayew und Venkatachalam 2012). Die Reaktion des Kapitalmarkts auf die positiven und negativen Signale wird mit Hilfe der abnormalen Rendite gemessen. Die Autoren finden eine positive (negative) abnormale Rendite bei einer positiven (negativen) emotionalen Stimmungslage.

Als eine Alternative zur Vorenthaltung von Informationen, können Manager auch auf vorformulierte Antworten zurückgreifen und so ungewollte Aussagen vermeiden. Die Studie von Lee (2016) legt eine negative Reaktion des Kapitalmarkts auf vorformulierte Aussagen nahe, allerdings werden die Informationen nicht unmittelbar vollumfänglich im Aktienkurs eingepreist, sodass das Ziel der Manager zumindest teilweise erreicht wird. Zudem findet der Autor Hinweise darauf, dass die vermehrte Verwendung vorformulierter Antworten auf unerwartete negative Ergebnisse hindeutet und der Informationsgehalt von Conference Calls durch weniger Spontanität sinkt (Lee 2016).

Auch die textuelle Stimmung in der Kommunikation innerhalb von Conference Calls kann weitreichende Implikationen haben. Da im Diskussionsteil Manager und Analysten sprechen, untersuchen beispielsweise Brockman et al. (2015), ob sich das textuelle Sentiment zwischen beiden Gruppen unterscheidet und inwieweit Investoren darauf reagieren. Die Ergebnisse deuten darauf hin, dass Manager nicht in der Lage sind, den optimistischen textuellen Tonfall der Präsentation in den Diskussionsteil zu transferieren und der Tonfall der Analysten generell pessimistischer ist als der von Managern. Die abnormale Rendite während des Conference Calls weist zudem darauf hin, dass Investoren dem Tonfall der Analysten mehr Beachtung schenken (Brockman et al. 2015). In ähnlicher Weise finden Chen et al. (2018) Indizien dafür, dass Investoren insbesondere auf die generell neutralere Analystenstimmung und weniger auf die vom Management vermittelte Stimmung reagieren. Übereinstimmend hiermit finden Milian und Smith (2017) einen positiven Effekt von lobenden Worten der Analysten auf die zukünftige abnormale Rendite. Eine Studie von Price et al. (2012) zeigt durch Inhaltsanalysen von Conference Call Protokollen einen signifikanten Zusammenhang zwischen dem textuellen Sentiment und der abnormalen Aktienrendite zum Zeitpunkt der Ergebnisbekanntgabe, dem postearnings-announcement drift und dem abnormalen Handelsvolumen.

Die Auswirkung der textuellen Stimmung kann jedoch auch von den Adressaten abhängen. So legt die Studie von Blau et al. (2015) nahe, dass die Reaktion von der Qualifikation der Investoren abhängt. Leerverkäufer als qualifizierte Investoren beziehen die im Conference Call übermittelten Informationen generell in ihre Aktivitäten mit ein. Dabei finden die Autoren Indizien dafür, dass andere Investoren gegenüber Leerverkäufern auf einen abnormalen Ton in Kombination mit einer Überschreitung der Analystenerwartung zu stark reagieren. Qualifizierte Investoren mit einer besseren Informationsverarbeitung interpretieren den übertriebenen Ton zumeist als negative Information. Während die meisten Studien, die den textuellen Ton untersuchen, mit einer simplen Relation von z. B. negativen bzw. positiven Wörtern zu den Gesamtwörtern als Stimmungsmaß arbeiten, nehmen Allee und 
DeAngelis (2015) hingegen an, dass auch die temporäre Verteilung der Stimmung von Bedeutung ist. Sie zeigen, dass die negative (positive) Reaktion von Analysten und Investoren umso stärker wird, je weiter die negative (positive) Stimmung innerhalb des Conference Calls gestreut ist. Zudem stellen die Autoren fest, dass die Verteilung des textuellen Tons mit der Erfüllung von Analystenschätzungen, den Offenlegungsentscheidungen sowie den Anreizen und Darstellungsweisen des Managements zusammenhängt.

Da die meisten hier erwähnten Studien im Bereich der Textanalyse mit Wortlisten arbeiten, ist deren Konstruktvalidität zur Messung von Stimmungen zu hinterfragen. Regelmäßig stammen die verwendeten Wörterbücher aus anderen Fachrichtungen (z. B. Psychologiewörterbuch Harvard-IV) und beinhalten keine finanzspezifischen Wörter. Die Anwendung allgemeiner Wörterbücher auf finanzspezifische Texte kann nach Henry und Leone (2016) sowie Loughran und McDonald (2011) zu fehlerhaften Ergebnissen führen. Selbst die Verwendung von finanzspezifischen Wörterbüchern ist kritisch zu prüfen, da sich Conference Calls gerade durch eine freie und interaktive Kommunikation auszeichnen. Neuere Studien thematisieren dieses Problem bewusst (Allee und DeAngelis 2015) und nehmen eine kontextspezifische Anpassung der Wortlisten von Loughran und McDonald (2011) vor. Andere Studien verwenden Wortlisten, deren Anzahl der positiven bzw. negativen Wörter unausgeglichen oder extrem gering sind.

Auch die ökonomische Wirkung sprachlicher Komplexität in Conference Calls erlangt eine erhöhte Aufmerksamkeit in der Literatur. Brochet et al. (2016) untersuchen beispielsweise die Auswirkung von Sprachbarrieren auf die Komplexität und Informationsverarbeitung am Kapitalmarkt. Dazu werden englische Conference Call Protokolle von Unternehmen aus nicht englischsprachigen Ländern untersucht. Die Autoren finden heraus, dass Sprachbarrieren die Transparenz im Rahmen von Conference Calls vermindern, indem Manager zu komplexen Formulierungen neigen oder falsche Ausdrücke verwenden. Hieraus resultieren schwächere Kapitalmarktreaktionen, was die Autoren auf eine verminderte Informationsqualität aufgrund von Sprachbarrieren zurückführen.

Die bisher vorgestellten Studien befassen sich ausschließlich mit den Kapitalmarktwirkungen von Unternehmen, die selbst Conference Calls durchführen. Dabei liegt die Vermutung nahe, dass sich auch für Unternehmen derselben Branche, die ähnlichen makroökonomischen, aber auch branchenspezifischen Chancen und Risiken unterliegen (Peer-Unternehmen), Auswirkungen ergeben. Dieses Phänomen zeigt sich empirisch in der Parallelbewegung der Aktienkurse aller Peer-Unternehmen im Falle der Offenlegung neuer Informationen von einem dieser Unternehmen. Brochet et al. (2018) untersuchen dieses Phänomen erstmals für Conference Calls. Die Autoren stellen fest, dass die Parallelbewegung nach Conference Calls stärker ausgeprägt ist als nach Ergebnisveröffentlichungen. Als einen wesentlichen Treiber dieses Effekts identifizieren Brochet et al. (2018) die Rolle von Analysten. So können sell-side Analysten, die sich auf eine Branche spezialisieren und/oder mehrere PeerUnternehmen gleichzeitig abdecken, Auswirkungen von neuen Informationen auf Peer-Unternehmen besser abschätzen und effizient an ihre Kunden kommunizieren. Mit der Vermutung übereinstimmend ist das beobachtete Phänomen besonders stark 
ausgeprägt, wenn sell-side Analysten sowohl das Conference Call durchführende Unternehmen als auch die Peer-Unternehmen abdecken.

Eine wesentliche Herausforderung der untersuchten Studien liegt in der Identifikationsstrategie der festgestellten Effekte, da Conference Calls zumeist unmittelbar nach Veröffentlichung der Quartals- oder Jahresabschlüsse erfolgen. Studien, die Renditen auf Tagesbasis ermitteln, können letztlich nicht eindeutig separieren, ob sich Kapitalmarkteffekte auf die Informationen aus dem Abschluss oder jene aus dem Conference Call beziehen. Daher greifen neuere Studien vermehrt auf Intraday-Daten zurück (z. B. Brochet et al. 2018). Diese Vorgehensweise ermöglicht eine klarere Separierung von Kapitalmarkteffekten von Conference Calls versus Ergebnisveröffentlichungen.

Offene Forschungsfragen Zukünftige Forschungsarbeiten könnten eine differenziertere Betrachtung der Wirkung einzelner Teilnehmer vornehmen. Die bisherigen Studien, die sich mit Conference Call Transkripten aus nicht englischsprachigen Ländern beschäftigen, gehen beispielsweise nicht auf die mögliche Problematik der Sprachfähigkeiten der Fragenden ein. So sind unter den an deutschen Conference Calls teilnehmenden Analysten regelmäßig sowohl nationale als auch internationale Vertreter zu finden. Durch die unterschiedliche Sprachkompetenz der Teilnehmer können Sprachbarrieren bzw. -problematiken hervorgerufen werden, welche das Kommunikationsverhalten beeinträchtigen. Folglich könnte der Kapitalmarkt auf Fragen von Teilnehmern mit verschieden ausgeprägter Sprachkompetenz unterschiedlich reagieren. In ähnlicher Weise könnte die Stärke der Reaktion ebenfalls von der Art (buy-side oder sell-side), der Qualifikation oder dem Ansehen und weiteren Eigenschaften des Fragenden abhängig sein.

\subsubsection{Analystenschätzungen}

Die folgenden Studien analysieren den Einfluss von Conference Calls auf die Arbeit von Analysten. Bowen et al. (2002) zeigen, dass sowohl der Vorhersagefehler als auch die Streuung der Analystenvorhersagen in Quartalen mit Conference Calls geringer sind als in Quartalen ohne. Die Autoren sehen dies als Indiz dafür, dass durch Conference Calls zusätzliche Informationen veröffentlicht werden. Davon profitieren insbesondere Analysten mit weniger stark ausgeprägten Prognosefähigkeiten. Für den deutschen Kapitalmarkt kommen Bassemir et al. (2013) in einer vergleichbaren Studie zu ähnlichen Ergebnissen. Die Vorhersagefehler reduzieren sich jedoch stärker, was die Autoren auf das weniger strenge Prognoseumfeld zurückführen. Zudem konnte keine Veränderung der Streuung der Analystenvorhersagen festgestellt werden. Dieses Ergebnis sehen die Autoren als Resultat aus zwei gegenläufigen Effekten. Einerseits aus dem Rückgang der allgemeinen Unsicherheit und andererseits aus der Zunahme der Informationsasymmetrien zwischen den Analysten durch neue, private Informationen (Bassemir et al. 2013).

Kimbrough (2005) untersucht, ob Analysten durch Conference Calls aktuelle Ergebnisinformationen für Prognosen effizienter nutzen können. Die Ergebnisse zeigen eine sinkende Autokorrelation der Vorhersagefehler bei Unternehmen mit Confe- 
rence Calls. Dies ist ein Anzeichen dafür, dass Analysten durch Conference Calls schneller auf zukunftsbezogene Informationen reagieren.

Kross und Suk (2012) nehmen an, dass Analysten nach Einführung der Reg FD nur noch Informationen durch die öffentliche Berichterstattung der Unternehmen erhalten und kein Zugang zu privaten Informationen besteht. Damit stehen allen Marktteilnehmern zum Zeitpunkt der Veröffentlichung die Informationen gleichermaßen zur Verfügung. Sie finden Hinweise dafür, dass sich nach der Gesetzesänderung die Geschwindigkeit und Anzahl von Änderungen der Analystenprognosen erhöht und sich deren Qualität durch den Fehler- und Streuungsrückgang verbessert hat. Die Relevanz der Reg FD zeigt auch Irani (2004), dessen Ergebnisse darauf hindeuten, dass Conference Calls nach der Regulierung mehr relevante Informationen beinhalten und so die Übereinstimmung und Genauigkeit der Analystenprognosen erhöhen.

Im Rahmen von Conference Calls gibt es Analysten, die teilnehmen und sich aktiv einbringen und solche, die dies nicht tun. Mayew et al. (2013) können zeigen, dass teilnehmende gegenüber nicht teilnehmenden Analysten genauere und zeitnähere Einschätzungen veröffentlichen. Dabei stellt sich die Frage, ob teilnehmende Analysten ihre privaten Informationen aus der Teilnahme am Conference Call gewinnen oder diese bereits zuvor besaßen. Wenn Manager Conference Calls als Quelle von privat zugänglichen Informationen ansehen, dann vermuten Mayew et al. (2013), dass sich Analysten für den aktiven Zugang durch positive Aktienbewertungen empfehlen müssen. Diese Vermutung kann allerdings nicht nachgewiesen werden.

Eine weitere Studie analysiert die Auswirkungen von Lob der Analysten an das Management mit Hilfe von Wortlisten. Milian et al. (2017) finden in ihrer Studie einen positiven Zusammenhang zwischen der Prognosegenauigkeit von Analysten und deren Sprachfärbung innerhalb von Conference Calls. Einen möglichen Grund für diese Beziehung sehen die Autoren im besseren Zugang zu privaten Informationen des Managements für wohlgesonnene Analysten.

Frankel et al. (2017) zeigen in einem aktuellen Arbeitspapier einen neuen Ansatz zur Messung der für Analysten verwertbaren Information durch neue (teil-)automatisierte Verfahren. Die Autoren nutzen dabei insgesamt vier Textanalyseverfahren, um aus den Protokollen von Conference Calls zukünftig revidierte Analystenschätzungen zu prognostizieren und so die Nützlichkeit der qualitativen Informationen zu messen. Drei Verfahren (support vector regressions, supervised LDA sowie random forest regression trees) des maschinellen Lernens versuchen jeweils auf unterschiedliche Weise den Inhalt von Texten zu extrahieren. Zudem wird der textuelle Ton auf Basis von Wortlisten hinzugezogen. Die Autoren kombinieren die Maße zu einem Gesamtmaß und finden eine hohe Erklärungskraft für das Auftreten einer revidierten Analystenprognose im Folgequartal. Diese kann jedoch weiter gesteigert werden, wenn erstens extrem seltene Wörter aus der Berechnung eliminiert werden und zweitens, wenn der unternehmensindividuelle oder marktspezifische Kontext in der Schätzung berücksichtigt wird. In gleicher Weise kann die Anwendung von linguistischen Netzen, wie etwa Clusteranalyse (Feldman und Sanger 2007), von Assoziationsnetzen (Feldman und Sanger 2007), GABEK-Netzen (Zelger 2000; Raich 
et al. 2014) oder ähnlichen Mixed-Methods Verfahren, die Textanalysemöglichkeiten von Conference Calls erweitern und neue Zusammenhänge aufdecken.

Offene Forschungsfragen Die oben erläuterten Studien fokussieren sich größtenteils auf die unmittelbare Performance von Analysten bei Teilnahme an einem Conference Call. Eine detaillierte Untersuchung des Informationsbeschaffungsprozesses nimmt bisher keine Studie vor. Ein lohnenswerter Ansatz wäre deshalb eine Untersuchung der Informationsbeschaffungskanäle von Analysten. So ist einerseits unklar, welche Informationen aus Conference Calls und welche aus anderen Quellen stammen. Folglich könnte sich entweder eine substituierende oder eine ergänzende Beziehung zwischen Conference Calls und anderen Informationsbeschaffungsmöglichkeiten (wie etwa persönliche Treffen mit dem Management) ergeben. Andererseits ist wenig über die Informationsbeschaffung aus der Teilnahme an mehreren Conference Calls bekannt. So wurde bislang nicht untersucht, ob Analysten, die mehrere Unternehmen innerhalb einer bestimmten Branche abdecken, gleiche Fragen bei unterschiedlichen Veranstaltungen stellen, um die Aussagen von Managern zu Märkten, Produkten und Trends zu verifizieren. Alternativ könnten sich Analysten durch verschiedene Fragen in unterschiedlichen Conference Calls ein breiteres Bild (beispielsweise über Branchentrends) verschaffen und so Informationsvorteile erzielen. Insgesamt könnten zukünftige Forschungsvorhaben wichtige Erkenntnisse dazu liefern, welche Informationen aus Conference Calls stammen, welche Strategien Analysten zur Beschaffung der Informationen verfolgen und in welchem Maß diese Faktoren deren Prognosen beeinflussen.

\subsubsection{Kapitalkosten}

Zwei im Rahmen dieser Arbeit vorgestellte Studien erforschen den Zusammenhang zwischen Conference Calls und Kapitalkosten. Brown et al. (2004) untersuchen, ob die Einführung und das regelmäßige Ausrichten von Conference Calls zu einer Reduzierung von Informationsasymmetrien bei Investoren und damit zu niedrigeren Kapitalkosten für Unternehmen führen. Francis et al. (2008) untersuchen in einer breiter angelegten Studie den Zusammenhang von freiwilliger Berichterstattung, Ergebnisqualität und Kapitalkosten.

Brown et al. (2004) stellen bei einer regelmäßigen Durchführung von Conference Calls eine Senkung des durchschnittlichen Levels der Informationsasymmetrien fest, was eine Reduzierung der Kapitalkosten nach sich zieht. Dies deutet darauf hin, dass Unternehmen erst bei einer längerfristigen Entscheidung zugunsten einer umfangreicheren Informationspolitik davon profitieren (Brown et al. 2004). Zu gegensätzlichen Ergebnissen kommen jedoch Francis et al. (2008). Zwar führen mehr freiwillige Veröffentlichungen grundsätzlich zu geringeren Kapitalkosten, allerdings nicht bei Conference Calls. Das Ergebnis führen Francis et al. (2008) auf die unterschiedlichen Veröffentlichungsformen zurück, da diese qualitativ verschiedene Inhalte aufweisen und unterschiedlichen Managementzielen dienen können.

In einem aktuellen Arbeitspapier extrahieren Donovan et al. (2018) mit Hilfe von Methoden des maschinellen Lernens (support vector regressions, supervised LDA sowie random forest regression trees) qualitative Informationen zum Kreditrisiko aus 
Conference Calls. Die Autoren finden eine hohe Erklärungskraft für die Variation der Spreads von Credit Default Swaps (CDS) durch einen aggregierten Wert aus den drei verwendeten Methoden. Für Unternehmen ohne aktiv gehandelte CDS zeigt sich ein hoher Erklärungsgehalt für sonstige Kreditwürdigkeitsindikatoren, wie etwa künftige Ratingabstufungen oder Änderungen in den Zinsspreads.

Offene Forschungsfragen Auf Basis der bisherigen Erkenntnisse ist der Effekt von Conference Calls auf Kapitalkosten nicht eindeutig. Ein besseres Verständnis über den Zusammenhang bietet möglicherweise eine textuelle Auswertung der Protokolle. Durch die Analyse von Finanzberichten zeigen beispielsweise Bonsall und Miller (2017), dass Fremdkapitalgeber negativ auf schwer verständliche Unternehmenspublikationen reagieren. So könnte die unzureichende oder schwer verständliche Beantwortung der kreditrisikorelevanten Fragen in Conference Calls zu schlechteren Kreditkonditionen führen. In ähnlicher Weise könnte sich eine präzise Beantwortung der Fragen und die Preisgabe von relevanten Informationen positiv auf die Refinanzierungskonditionen auswirken.

\subsection{Conference Calls als Informationsquelle für andere Forschungsfragen}

In Abgrenzung zu den bisher vorgestellten Studien anhand der Kategorien unseres Rahmenkonzepts sind zudem Beiträge in der Literatur zu finden, welche die einzigartigen Charakteristika von Conference Calls nutzen, um Rückschlüsse auf offene Forschungsfragen aus angrenzenden Bereichen wie der Rechnungslegungsqualität oder dem Managementverhalten zu ziehen. Diese werden im Folgenden kurz vorgestellt.

\subsubsection{Rechnungslegungsqualität und Unternehmensbewertung}

Hobson et al. (2012) untersuchen, inwieweit die Analyse von Sprache im Rahmen von Conference Calls verwendet werden kann, um falsche bzw. fehlerhafte Berichterstattung zu identifizieren. Es wird angenommen, dass die Wahrscheinlichkeit von falscher Berichterstattung positiv mit stimmlichen Anzeichen für kognitive Dissonanz zusammenhängt. Hobson et al. (2012) führen eine Sprachanalyse von Conference Call Audiomitschnitten mittels LVA-Software durch und können nachträgliche Fehlerkorrekturen in der Finanzberichterstattung mit einer höheren Wahrscheinlichkeit vorhersagen. Ein ähnliches Ziel verfolgen Larcker und Zakolyukina (2012), die der Frage nachgehen, ob es durch die Analyse von Managementaussagen möglich ist, betrügerisches Handeln aufzudecken. Hintergrund ist die Annahme, dass CEOs und CFOs im Falle einer manipulierten Finanzberichterstattung Kenntnis davon haben und diese Kenntnis zu einer veränderten beobachtbaren Ausdrucksweise führt. Das auf Wortlisten basierende Modell führt zu einer höheren Entdeckungswahrscheinlichkeit einer fehlerhaften Berichterstattung. Somit erzielen sowohl die Modelle von Hobson et al. (2012) als auch die von Larcker und Zakolyukina (2012) eine bessere oder zumindest gleichwertige Prognosequalität im Vergleich zu bestehenden, auf Finanzzahlen basierenden Modellen. 
Wie unterschiedlich die Wahrnehmungen von bilanzpolitischen Instrumenten sein können, untersuchen Hope und Wang (2018) anhand von Big Bath Accounting. Sie zeigen, dass die Durchführung einer solchen Maßnahme mit einer signifikanten Erhöhung der Informationsasymmetrien korrespondiert, wenn das bilanzpolitische Instrument durch einen irreführenden statt eines ehrlichen CEO durchgeführt wird. Zur Identifikation von ehrlichen bzw. irreführenden Managern bedienen die Autoren sich der Textanalyse (ähnlich wie Larcker und Zakolyukina 2012). Daraus folgern die Autoren, dass der Markt identifizieren kann, welche Manager ehrlich bzw. irreführend sind.

Auch die Kommunikationsweise des Managements kann Hinweise auf die Anwendung von Bilanzpolitik geben. Auf Basis dessen identifizieren Capalbo et al. (2018) beispielsweise den Narzissmus des Managements und untersuchen den Einfluss dieser Charaktereigenschaft auf die Rechnungslegungsqualität. Die Operationalisierung für Narzissmus erfolgt durch die Bildung der Relation von Personalpronomen der ersten Person Singular gegenüber Personalpronomen der ersten Person Singular und Plural in den Aussagen des Managements. Dieser Wert weist eine signifikant positive Beziehung zur Höhe der diskretionären Periodenabgrenzungen auf. In ähnlicher Weise zeigen Brochet et al. (2015), dass sich in verschiedenen Facetten des Kommunikationsverhaltens Hinweise auf die kurzfristige Maximierung bestimmter ökonomischer Kennzahlen finden.

Die Kommunikation innerhalb von Conference Calls könnte außerdem einen Einfluss auf die Bewertungsunsicherheit des durchführenden Unternehmens haben. So finden Borochin et al. (2018) einen negativen Zusammenhang zwischen der Grundstimmung eines Conference Calls und der Bewertungsunsicherheit des Unternehmens auf dem Markt für Eigenkapitalinstrumente.

\subsubsection{Managementverhalten und -charakteristika}

Eine Reihe von Studien analysiert bestimmte Verhaltensweisen und Persönlichkeitsmerkmale des Managements auf Basis ihrer Kommunikation in Conference Calls. Li et al. (2014) nutzen Conference Call Protokolle, um das Kommunikationsverhalten von Managern zu untersuchen. Dabei gehen sie der Frage nach, wo das Wissen innerhalb des Managements zu finden ist und welche Auswirkungen dies auf die Gehälter bzw. den Unternehmenswert hat. Die Ergebnisse deuten darauf hin, dass das Kommunikationsverhalten während eines Conference Calls nicht willkürlich, sondern von der Verteilung des spezifischen Wissens innerhalb des Managements abhängig ist. Da der Wissensstand nicht beobachtbar ist, dienen insbesondere Maße des Bildungsgrades als Approximation. Der Redeanteil des CEOs steigt bei besserer Ausbildung und Innehaben einer zusätzlichen, zertifizierten Berufsqualifikation im Rechnungswesen (z.B. CPA). Im Gegensatz dazu sinkt der Anteil, wenn der CEO neu ist oder das Unternehmen hohe Forschungsaufwendungen hat. Hinsichtlich des Zusammenhangs zwischen dem Kommunikationsumfang des CEOs und dessen Vergütung zeigt die Studie, dass CEOs mit höherem Redeanteil auch eine höhere Vergütung erhalten. Zudem weisen Unternehmen, die Manager mit aktiver Kommunikation besser vergüten, einen höheren Marktwert auf (Li et al. 2014). 
Die in Conference Calls vermittelte Stimmung könnte neben den Handlungen der Investoren auch die des Managements beeinflussen. Wie die Studie von Brockman et al. (2017) zeigt, betreiben Manager des durchführenden Unternehmens einen dem Ton des Conference Calls entgegengesetzten Handel mit eigenen Aktien. Daraus folgern die Autoren, dass die hauptsächlich für den Ton verantwortlichen Manager entgegen ihrer eigens vermittelten Stimmung handeln. Manager beziehen in ihre Handlungsentscheidung wohl auch die Aussagen von Analysten mit ein, indem sie ihre Aktienverkäufe verstärken (verringern), wenn der Analystenton im Q\&A-Teil (weniger) pessimistisch wird.

In diesem Kontext zeigen Chapman und Green (2018) jedoch auch, dass Analysten durch Nachfragen das Managementverhalten beeinflussen können. So sind Manager, denen konkrete zukunftsbezogene Anfragen zu Rechnungslegungsthematiken gestellt werden, eher dazu geneigt, zukünftig konkrete Informationen zu diesen Themen zur Verfügung zu stellen. Dieser Effekt sinkt im Zeitverlauf und ist besonders stark ausgeprägt bei Unternehmen mit schwachem Informationsumfeld. Die Autoren schlussfolgern, dass das Management die Informationsbedürfnisse der Analysten aus Conference Calls ableitet und die Kommunikation entsprechend darauf abstimmt.

\section{Zusammenfassung}

Zusammenfassend sind Conference Calls nach zwei Jahrzehnten weiterhin im Fokus der internationalen Forschung. Der vorliegende Beitrag stellt den aktuellen Stand der empirischen Forschung anhand eines Rahmenkonzepts systematisch dar. Hierzu werden die Determinanten und ökonomischen Konsequenzen von Conference Calls diskutiert. Zudem werden Studien näher analysiert, welche das Phänomen Conference Call als Offenlegungsinstrument für eine Vielzahl von weiteren Themensträngen wie den Informationsgehalt sowie das Kommunikationsverhalten des Managements untersuchen. Die Ergebnisse unserer Literaturanalyse sind sowohl für deutsche Unternehmen als auch für Rechnungslegungsadressaten von hoher Relevanz, da insbesondere in Deutschland kapitalmarktorientierte Unternehmen den Zugang zu Conference Calls sehr unterschiedlich ausüben. Die Bemühungen des europäischen Gesetzgebers zu einer stärkeren Transparenz am Kapitalmarkt schließen aktuell eine Verpflichtung zur offenen Ausübung von Conference Calls (noch) nicht ein.

Unsere Literaturanalyse identifiziert auch eine Vielzahl an offenen Forschungsfragen, welche für den wissenschaftlichen Adressatenkreis von Interesse sein sollten. Ungeklärt ist in der Literatur beispielsweise die Frage, inwiefern Kapitalmarktteilnehmer ihre Informationsinteressen einerseits durch Conference Calls, andererseits aber auch durch private Treffen mit dem Management befriedigen. Ferner bietet das Forschungsfeld zahlreiche Schnittpunkte zu angrenzenden Disziplinen, etwa der Psychologie und der Managementforschung. Zudem ermöglicht die zunehmende Verbreitung und Verfügbarkeit von (Audio-)Mitschnitten und Protokollen mit Hilfe neuer Software sowie computergestützter Textanalysemethoden eine methodische Weiterentwicklung der Conference Call Literatur. Dabei weisen wir auf aktuelle 
Ansätze hin, welche die empirische Kapitalmarktforschung zukünftig noch stärker prägen werden.

Danksagung Die Autoren bedanken sich bei Christian Dürholt, Petroula Glachtsiou, Klaus Henselmann, Anna Nowotsch, Thorsten Sellhorn (Associate Editor), Oliver Voß, Alfred Wagenhofer (Editor) sowie zwei anonymen Gutachtern für wertvolle Anmerkungen.

Funding Open Access funding provided by Projekt DEAL.

Open Access Dieser Artikel wird unter der Creative Commons Namensnennung 4.0 International Lizenz veröffentlicht, welche die Nutzung, Vervielfältigung, Bearbeitung, Verbreitung und Wiedergabe in jeglichem Medium und Format erlaubt, sofern Sie den/die ursprünglichen Autor(en) und die Quelle ordnungsgemäß nennen, einen Link zur Creative Commons Lizenz beifügen und angeben, ob Änderungen vorgenommen wurden.

Die in diesem Artikel enthaltenen Bilder und sonstiges Drittmaterial unterliegen ebenfalls der genannten Creative Commons Lizenz, sofern sich aus der Abbildungslegende nichts anderes ergibt. Sofern das betreffende Material nicht unter der genannten Creative Commons Lizenz steht und die betreffende Handlung nicht nach gesetzlichen Vorschriften erlaubt ist, ist für die oben aufgeführten Weiterverwendungen des Materials die Einwilligung des jeweiligen Rechteinhabers einzuholen.

Weitere Details zur Lizenz entnehmen Sie bitte der Lizenzinformation auf http://creativecommons.org/ licenses/by/4.0/deed.de.

Interessenkonflikt D. Kaya, C. Maier und T. Böhmer geben an, dass kein Interessenkonflikt besteht.

\section{Literatur}

Allee, Kristian D., und Matthew D. DeAngelis. 2015. The structure of voluntary disclosure narratives: Evidence from tone dispersion. Journal of Accounting Research 53:241-274.

Bassemir, Moritz, Zoltan Novotny-Farkas, und Julian Pachta. 2013. The effect of conference calls on analysts' forecasts - German evidence. European Accounting Review 22:151-183.

Bernard, Victor L., und Jacob K. Thomas. 1989. Post-earnings-announcement drift: Delayed price response or risk premium? Journal of Accounting Research 27:1-36.

Bernard, Darren, David Burgstahler, und Devrimi Kaya. 2018a. Size management by European private firms to minimize proprietary costs of disclosure. Journal of Accounting and Economics 66:94-122.

Bernard, Darren, Devrimi Kaya, und John Wertz. 2018b. Entry and capital structure mimicking in concentrated markets: The role of incumbents' financial disclosures. Working Paper.

Beyer, Anne, Daniel A. Cohen, Thomas Z. Lys, und Beverly R. Walther. 2010. The financial reporting environment: Review of the recent literature. Journal of Accounting and Economics 50:296-343.

Bischof, Jannis, Holger Daske, und Christoph Sextroh. 2014. Fair value-related information in analysts' decision processes: Evidence from the financial crisis. Journal of Business Finance and Accounting 41:363-400.

Blau, Benjamin M., Jared R. DeLisle, und S. McKay Price. 2015. Do sophisticated investors interpret earnings conference call tone differently than investors at large? Evidence from short sales. Journal of Corporate Finance 31:203-219.

Böhmer, Tobias, Oliver Voß, und Petroula Glachtsiou. 2019. Unternehmenskommunikation und Informationsbedürfnisse von Kapitalmarktteilnehmern - Eine empirische Analyse von Analystenkonferenzen der DAX30-Unternehmen. Zeitschrift für internationale und kapitalmarktorientierte Rechnungslegung (KoR) 19:394-399.

Bonsall, Samuel B., und Brian P. Miller. 2017. The impact of narrative disclosure readability on bond ratings and the cost of debt. Review of Accounting Studies 22:608-643.

Borochin, Paul A., James E. Cicon, Jared R. DeLisle, und S. McKay Price. 2018. The effects of conference call tones on market perceptions of value uncertainty. Journal of Financial Markets 40:75-91.

Botosan, Christine A., und Mary Stanford. 2005. Managers' motives to withhold segment disclosures and the effect of SFAS No. 131 on analysts' information environment. The Accounting Review 80:751-772. 
Bowen, Robert M., Angela K. Davis, und Dawn A. Matsumoto. 2002. Do conference calls affect analysts' forecasts? The Accounting Review 77:285-316.

Bowen, Robert M., Shantanu Dutta, Songlian Tang, und Pengcheng Zhu. 2018. Inside the "black box" of private in-house meetings. Review of Accounting Studies 23:487-527.

Bradshaw, Mark T. 2011. Analysts' forecasts: What do we know after decades of work? Working Paper.

Brochet, François, Kalin Kolev, und Alina Lerman. 2018. Information transfer and conference calls. Review of Accounting Studies 23:907-957.

Brochet, François, Maria Loumioti, und George Serafeim. 2015. Speaking of the short-term: Disclosure horizon and managerial myopia. Review of Accounting Studies 20:1122-1163.

Brochet, François, Gregory S. Miller, Patricia Naranjo, und Gwen Yu. 2019. Managers' cultural background and disclosure attributes. The Accounting Review 94:57-86.

Brochet, François, Patricia Naranjo, und Gwen Yu. 2016. The capital market consequences of language barriers in the conference calls of Non-U.S. firms. The Accounting Review 91:1023-1049.

Brockman, Paul, James E. Cicon, Xu Li, und S. McKay Price. 2017. Words versus deeds: Evidence from post-call manager trades. Financial Management 46:965-994.

Brockman, Paul, Xu Li, und S. McKay Price. 2015. Differences in conference call tones: Managers vs. analysts. Financial Analysts Journal 71:24-42.

Brown, Stephen, Stephen A. Hillegeist, und Kin Lo. 2004. Conference calls and information asymmetry. Journal of Accounting and Economics 37:343-366.

Bushee, Brian J., Ian D. Gow, und Daniel J. Taylor. 2018. Linguistic complexity in firm disclosures: Obfuscation or information? Journal of Accounting Research 56:85-121.

Bushee, Brian J., Dawn A. Matsumoto, und Gregory S. Miller. 2003. Open versus closed conference calls: The determinants and effects of broadening access to disclosure. Journal of Accounting and Economics 34:149-180.

Bushee, Brian J., Dawn A. Matsumoto, und Gregory S. Miller. 2004. Managerial and investor responses to disclosure regulation: The case of Reg FD and conference calls. The Accounting Review 79:617-643.

Call, Andrew C., Nathan Y. Sharp, und Thomas D. Shohfi. 2018. Which buy-side institutions participate in public earnings conference calls? Implications for capital markets and sell-side coverage. Working Paper.

Capalbo, Francesco, Alex Frino, Ming Y. Lim, Vito Mollica, und Riccardo Palumbo. 2018. The impact of CEO narcissism on earnings management. Abacus 54:210-226.

Chan, Ann L.-C., Edward Lee, Jirada Petaibanlue, und Ning Tan. 2017. Do board interlocks motivate voluntary disclosure? Evidence from Taiwan. Review of Quantitative Finance and Accounting 48:441-466.

Chapman, Kimball, und Jeremiah R. Green. 2018. Analysts' influence on managers' guidance. The Accounting Review 93:45-69.

Chen, Jason V., Venky Nagar, und Jordan Schoenfeld. 2018. Manager-analyst conversations in earnings conference calls. Review of Accounting Studies 23:1315-1354.

Cheng, Qiang, Fei Du, Xin Wang, und Yutao Wang. 2016. Seeing is believing: Analysts' corporate site visits. Review of Accounting Studies 21:1245-1286.

Cicon, James. 2017. Say it again Sam: The information content of corporate conference calls. Review of Quantitative Finance and Accounting 48:57-81.

Davis, Angela K., Weili Ge, Dawn Matsumoto, und Jenny L. Zhang. 2015. The effect of manager-specific optimism on the tone of earnings conference calls. Review of Accounting Studies 20:639-673.

Dedman, Elisabeth, und Clive Lennox. 2009. Perceived competition, profitability and the withholding of information about sales and the cost of sales. Journal of Accounting and Economics 48:210-230.

Dell'Acqua, Alberto, Francesco Perrini, und Stefano Caselli. 2010. Conference calls and stock price volatility in the post-Reg FD era. European Financial Management 16:256-270.

Donovan, John, Jared Jennings, Kevin Koharki, und Joshua Lee. 2018. Determining credit risk using qualitative disclosure. Working Paper.

Feldman, Ronen, und James Sanger. 2007. The text mining handbook. Cambridge: Cambridge University Press.

Financial Accounting Standards Board (FASB). 2012. Disclosure framework: Invitation to comment. Norwalk: Financial Accounting Standards Board.

Francis, Jennifer, Dhananjay Nanda, und Per Olsson. 2008. Voluntary disclosure, earnings quality, and cost of capital. Journal of Accounting Research 46:53-99.

Frankel, Richard, Jared Jennings, und Joshua Lee. 2017. Using natural language processing to assess text usefulness to readers: The case of conference calls and earnings prediction. Working Paper. 
Frankel, Richard, Marilyn Johnson, und Douglas J. Skinner. 1999. An empirical examination of conference calls as a voluntary disclosure medium. Journal of Accounting Research 37:133-150.

Frankel, Richard, William J. Mayew, und Yan Sun. 2010. Do pennies matter? Investor relations consequences of small negative earnings surprises. Review of Accounting Studies 15:220-242.

Graham, John R., Campbell R. Harvey, und Shiva Rajgopal. 2005. The economic implications of corporate financial reporting. Journal of Accounting and Economics 40:3-73.

Heinrichs, Anne, Jihwon Park, und Eugene F. Soltes. 2019. Who consumes firm disclosures? - Evidence from earnings conference calls. The Accounting Review 94:205-231.

Henry, Elaine, und Andrew J. Leone. 2016. Measuring qualitative information in capital markets research. The Accounting Review 91:153-178.

Hobson, Jessen L., William J. Mayew, und Mohan Venkatachalam. 2012. Analyzing speech to detect financial misreporting. Journal of Accounting Research 50:349-392.

Hollander, Stephan, Maarten Pronk, und Erik Roelofsen. 2010. Does silence speak? An empirical analysis of disclosure choices during conference calls. Journal of Accounting Research 48:531-563.

Hope, Ole-Kristian, und Jingjing Wang. 2018. Management deception, big-bath accounting, and information asymmetry: Evidence from linguistic analysis. Accounting, Organizations and Society 70:33-51.

International Accounting Standards Board (IASB). 2017. Disclosure initiative_principles of disclosure. London: International Accounting Standards Board.

Irani, Afshad J. 2004. The effect of Regulation Fair Disclosure on the relevance of conference calls to financial analysts. Review of Quantitative Finance and Accounting 22:15-28.

Johnson, Travis L., und Eric C. So. 2018. Time will tell: Information in the timing of scheduled earnings news. Journal of Financial and Quantitative Analysis 53:2431-2464.

Jung, Michael J., M. H. Franco Wong, und X. Frank Zhang. 2018. Buy-side analysts and earnings conference calls. Journal of Accounting Research 56:913-952.

Kaya, Devrimi, und Andreas Seebeck. 2018. Computergestützte Textanalyse von erweiterten Bestätigungsvermerken. Die Wirtschaftsprüfung (WPg) 71:995-1002.

Ke Bin, und Kathy R. Petroni. 2004. How informed are actively trading institutional investors? Evidence from their trading behavior before a break in a string of consecutive earnings increases. Journal of Accounting Research 42:895-927.

Ke Bin, Kathy R. Petroni, und Yong Yu. 2008. The effect of Regulation FD on transient institutional investors' trading behavior. Journal of Accounting Research 46:853-883.

Kimbrough, Michael D. 2005. The effect of conference calls on analyst and market underreaction to earnings announcements. The Accounting Review 80:189-219.

Kross, William J., und Inho Suk. 2012. Does Regulation FD work? Evidence from analysts' reliance on public disclosure. Journal of Accounting and Economics 53:225-248.

Lang, Mark, und Russell Lundholm. 1993. Cross-sectional determinants of analyst ratings of corporate disclosures. Journal of Accounting Research 31:246-271.

Larcker, David F., und Anastasia A. Zakolyukina. 2012. Detecting deceptive discussions in conference calls. Journal of Accounting Research 50:495-540.

Leary, Mark T., und Michael R. Roberts. 2014. Do peer firms affect corporate financial policy? Journal of Finance 69:139-178.

Lee, Joshua. 2016. Can investors detect managers' lack of spontaneity? Adherence to predetermined scripts during earnings conference calls. The Accounting Review 91:229-250.

Lee, Chun I., Leonard Rosenthal, und Kimberly Gleason. 2004. Effect of Regulation FD on asymmetric information. Financial Analysts Journal 60:79-89.

Leuz, Christian, und Peter D. Wysocki. 2016. The economics of disclosure and financial reporting regulation: Evidence and suggestions for future research. Journal of Accouting Research 54:525-622.

Levy, Hagit, Ron Shalev, und Emanuel Zur. 2018. The effect of CFO personal litigation risk on firms' disclosure and accounting choices. Contemporary Accounting Research 35:434-463.

Li, Feng, Michael Minnis, Venky Nagar, und Madhav Rajan. 2014. Knowledge, compensation, and firm value: An empirical analysis of firm communication. Journal of Accounting and Economics 58:96-116.

Liang, Jia-Wen, Mai-Feng Lin, und Chen-Lung Chin. 2012. Does foreign institutional ownership motivate firms in an emerging market to increase voluntary disclosure? Evidence from Taiwan. Review of Quantitative Finance and Accounting 39:55-76.

Loughran, Tim, und Bill McDonald. 2011. When is a liability not a liability? Textual analysis, dictionaries, and 10-Ks. The Journal of Finance 66:35-65.

Loughran, Tim, und Bill McDonald. 2016. Textual analysis in accounting and finance: A survey. Journal of Accounting Research 54:1187-1230. 
Matsumoto, Dawn, Maarten Pronk, und Erik Roelofsen. 2011. What makes conference calls useful? The information content of managers' presentations and analysts' discussion sessions. The Accounting Review 86:1383-1414.

Mayew, William J. 2008. Evidence of management discrimination among analysts during earnings conference calls. Journal of Accounting Research 46:627-660.

Mayew, William J., und Mohan Venkatachalam. 2012. The power of voice: Managerial affective states and future firm performance. The Journal of Finance 67:1-43.

Mayew, William J., Nathan Y. Sharp, und Mohan Venkatachalam. 2013. Using earnings conference calls to identify analysts with superior private information. Review of Accounting Studies 18:386-413.

Milian, Jonathan A., und Antoinette L. Smith. 2017. An investigation of analysts' praise of management during earnings conference calls. Journal of Behavioral Finance 18:65-77.

Milian, Jonathan A., Antoinette L. Smith, und Elio Alfonso. 2017. Does an analyst's access to information vary with the favorableness of their language when speaking to management? Accounting Horizons 31:13-31.

Minnis, Michael, und Nemit Shroff. 2017. Why regulate private firm disclosure and auditing? Accounting and Business Research 47:473-502.

Price, S. McKay, James S. Doran, David R. Peterson, und Barbara A. Bliss. 2012. Earnings conference calls and stock returns: The incremental informativeness of textual tone. Journal of Banking \& Finance 36:992-1011.

Raich, Margit, Julia Müller, und Dagmar Abfalter. 2014. Hybrid analysis of textual data. Management Decision 52:737-754.

Securities and Exchange Commission (SEC). 2013. Report on review of disclosure requirements in regulation S-K. http://www.sec.gov/news/studies/2013/reg-sk-disclosure-requirements-review.pdf. Zugegriffen: 1. Okt. 2019.

Tasker, Sarah C. 1998a. Bridging the information gap: Quarterly conference calls as a medium for voluntary disclosure. Review of Accounting Studies 3:137-167.

Tasker, Sarah C. 1998b. Technology company conference calls: A small sample study. Journal of Financial Statement Analysis 4:6-15.

Verrecchia, Robert E. 1990. Information quality and discretionary disclosure. Journal of Accounting and Economics 12:365-380.

Zelger, Josef. 2000. Twelve steps of GABEK WinRelan. In GABEK II. Zur qualitativen Forschung, Hrsg. Renate Buber, und Josef Zelger, 205-220. Innsbruck: Studienverlag. 